\title{
Real time correlation functions and the functional renormalisation group
}

\author{
Jan M. Pawlowski ${ }^{1,2}$ and Nils Strodthoff ${ }^{1}$ \\ ${ }^{1}$ Institut für Theoretische Physik, Universität Heidelberg, Philosophenweg 16, 69120 Heidelberg, Germany \\ ${ }^{2}$ ExtreMe Matter Institute EMMI, GSI, Planckstr. 1, D-64291 Darmstadt, Germany
}

\begin{abstract}
We put forward a functional renormalisation group approach for the direct computation of real time correlation functions, also applicable at finite temperature and density. We construct a general class of regulators that preserve the space-time symmetries, and allows the computation of correlation functions at complex frequencies. This includes both imaginary time and real time, and allows in particular the use of the plethora of imaginary time results for the computation of real time correlation functions. We also discuss real time computation on the Keldysh contour with general spatial momentum regulators. Both set-ups give access to the general momentum and frequency dependence of correlation functions.
\end{abstract}

PACS numbers: 12.38.Aw, 11.10.Wx , 11.30.Rd, 12.38.Gc

\section{INTRODUCTION}

Real time correlation functions and most importantly real time propagators are the key ingredient to gain access to dynamical observables in strongly interacting quantum field theories. One prominent example is the single particle spectral function that is defined via the imaginary part of the retarded two-point function. It comprises information about the spectrum of the theory and in particular about resonances, and their fate at finite temperature and density. Single particle spectral functions also serve as an input for the calculation of transport coefficients via Kubo formulae in an approach put forward recently in [1, 2].

In strongly correlated regimes of the theory at hand already imaginary time computations are quite involved. A prominent example is QCD, where first principle approaches, both in the continuum and on the lattice, only provide numerical access to the low-energy hadronic regime with confinement and chiral symmetry breaking. For example, in continuum QCD elaborated approximations, including the implementation of symmetry constraints, and solving techniques have been developed for the qualitative and quantitative access to QCD correlation functions 3, 4. Moreover, in most cases correlation functions can only be computed numerically. Then, an analytic continuation from Euclidean (imaginary) spacetime to Minkowski space-time has to be carried out based on numerical Euclidean data. This reconstruction of real time correlation functions such as spectral functions using given numerical Euclidean data is usually done with Maximum Entropy methods, Padé approximants or similar reconstruction methods. However, these approaches imply a certain bias about the continuation, require very accurate Euclidean data, and hence represent a challenging conceptual and numerical problem.

The above reconstruction problem can only be overcome within a direct numerical real time computation. The most direct approach is a computation within a real time formulation of the theory. This entails that the nontrivial strongly correlated dynamics of the the- ory has to be accessed within such a real time approach. Even though possible in principle, this does not allow to utilise directly the plethora of results obtained in Euclidean space for example in the form of numerical input data. An alternative approach that naturally extends and utilises the Euclidean computation to real time correlation functions is the extension of the former to complex frequencies. In a functional approach this is, loosely speaking, based on the computation of Euclidean momentum loops at complex external frequencies. This includes both, imaginary frequencies or Euclidean spacetime and real frequencies or Minkowski space-time. Such an approach was put forward in the functional renormalisation group (FRG), [5 10, for recent work with Dyson Schwinger equations (DSE) see [11-15].

Here we put forward a general FRG-framework applicable in the presence of general regulators and for full frequency and momentum dependencies of the correlation functions. This is indispensable in situations with nontrivial quantum, thermal and medium corrections to the dispersion relations of the theory at hand. The latter includes a wide range of applications from condensed matter, heavy ion collisions to quantum gravity.

More specifically we aim at calculations of the hadron spectrum and real time observables in general within the framework of the fQCD collaboration 16. The fQCD collaboration works on a quantitative first-principle approach to continuum QCD within the FRG framework, see 3, 4, for first publications. On the one hand, the application to quark and gluon spectral functions requires an extension which goes beyond the widely used spatial regulator functions. On the other hand, we aim at a fully numerical procedure which is not tailored to specific regulator classes. It has to work for generalisations of standard regulators with exponential decay properties in order to make the problem numerically tractable.

A further long-term goal is the extension of imaginary time results, such as those obtained in the fQCD framework, to nonequilibrium situations. There, the dynamics singles out the time direction in the first place. This asks for approximation schemes and regulators that take into account the causality constraints and conservation laws 
at nonequilibrium. Related approximation schemes on the basis of the 2PI approach have e.g. been discussed in [17-19], causal (time) regulators have been introduced in 20] on the Keldysh contour. In higher dimensions the latter can be amended by a regulator in spatial momenta in a mixed representation. In the present work we discuss the properties of such generic spatial momentum regularisation and results for the single particle spectral functions.

The set-up for our approach is discussed in detail in the first section of the paper and demonstrated using the spectral functions in the $O(N)$ model as an illustrative example. The complementary second part addresses different approaches towards direct real time calculations, where the complications of Euclidean or Semi-Euclidean approaches as the ones from above due to the necessity of performing an analytic continuation are absent. Here we put forward the formalism for the calculation of spectral functions in a closed time path (CTP) framework.

\section{PROPAGATORS AND SINGLE PARTICLE SPECTRAL FUNCTIONS}

Real- and imaginary time propagators are limiting cases of the two-point correlation function or propagator with complex frequency $\omega \in \mathbb{C}$. The current framework is based on the Euclidean imaginary time quantum field theory and we denote $\omega=\omega_{\mathrm{E}}+\mathrm{i} \omega_{\mathrm{M}}$ with Euclidean frequency $\omega_{\mathrm{E}}$ and Minkowski frequency $\omega_{\mathrm{M}}$. In this section we discuss the numerical computation of correlation functions at complex frequencies from Euclidean loop integrals. In the present work, we concentrate on the important example of the single-particle spectral function, but the formulation also applies to higher correlation functions.

\section{A. Spectral functions from Euclidean correlation functions at complex frequencies}

In the following we put forward an approach for the analytical continuation of Euclidean correlation functions to complex frequencies. The continuation is chosen such that they reduce to the corresponding real time correlation functions at purely imaginary frequency. The appropriate continuation is discussed at the example of the propagator.

We start by defining a propagator $G(\omega, \vec{p})$ for complex frequencies $\omega=\omega_{\mathrm{E}}+\mathrm{i} \omega_{\mathrm{M}}$ as the uniquely defined analytic continuation of the real time Feynman propagator $G_{\mathrm{F}}$ to complex frequencies $\omega_{\mathrm{M}} \in \mathbb{C}$, i.e.

$$
\begin{aligned}
G\left(\mathrm{i} \omega_{\mathrm{M}}, \vec{p}\right): & =-G_{\mathrm{F}}\left(\omega_{\mathrm{M}}, \vec{p}\right) \\
& =\mathrm{i} \int \mathrm{d}^{4} x\langle\mathcal{T} \phi(x) \phi(0)\rangle_{c} e^{-\mathrm{i} \omega_{\mathrm{M}} x^{0}+\mathrm{i} \vec{x} \vec{p}},
\end{aligned}
$$

where $\mathcal{T}$ denotes time ordering and the subscript indicates the connected two-point correlation function. Eq. (1) also holds true at finite temperature and density. Then the real time retarded propagator can be obtained from

$$
G_{\mathrm{R}}\left(p_{0}, \vec{p}\right)=-\lim _{\epsilon \rightarrow 0} G\left(-\mathrm{i}\left(p_{0}+\mathrm{i} \epsilon\right), \vec{p}\right),
$$

that is from the propagator evaluated at the complex momentum $\epsilon-\mathrm{i} p_{0}$ with $\omega_{\mathrm{E}}=\epsilon$ and $\omega_{\mathrm{M}}=-p_{0}$. The imaginary part of the retarded propagator relates directly to the spectral function,

$$
\rho(p)=-2 \operatorname{Im} G_{\mathrm{R}}(p),
$$

see B6. Evaluated at Euclidean frequencies $\omega=2 \pi n T$ with $n \in \mathbb{Z}$ the continuation $G$ defined in (1) coincides with the Euclidean Feynman propagator, $G(\omega, \vec{p})=$ $G_{\mathrm{E}}(\omega, \vec{p})$, which is defined via

$$
G_{\mathrm{E}}(\omega, \vec{p}):=\int_{0}^{\beta} \mathrm{d} x^{0} \int \mathrm{d}^{3} x\langle\mathcal{T} \phi(x) \phi(0)\rangle_{c} e^{\mathrm{i}\left(\omega x_{0}+\vec{p} \vec{x}\right)} .
$$

Note that at finite temperature the Euclidean propagator (4) is only defined for discrete values $\omega=2 \pi n T$ with $n \in \mathbb{Z}$. Therefore, there is no unique analytic continuation to $\omega \in \mathbb{C}$. A perfectly well-defined continuation is defined by taking (4) for $\omega \in \mathbb{R}$ on the whole real axis which has a unique continuation to $\omega \in \mathbb{C}$. The continuation defined in this way is a straightforward one in a Euclidean framework, and can be applied to lattice correlation functions in position space.

However, this continuation does not coincide with the real time propagator at complex momenta. Moreover, in continuum frameworks quantum corrections to correlation functions are computed from loop representations in frequency and momentum space, e.g. in perturbation theory and in particular in the method used in the present work, the FRG. Then, the frequency routing is non-unique for $\omega \neq 2 \pi n T$. This ambiguity can be used to our advantage in order to define the continuation to complex frequencies in such a way, that the difference to real time correlation functions is easily tracked down. Indeed, for any loop representation in frequency space the difference can be written as a sum over residues of the poles that depend on the external frequency, as well as potential cuts. For the Feynman propagator this reads

$$
G_{\mathrm{F}}\left(\omega_{\mathrm{M}}\right)=-G_{\mathrm{E}, \mathrm{cont}}\left(\mathrm{i} \omega_{\mathrm{M}}\right)+\sum_{\text {poles }} \text { Res }+ \text { Cuts },
$$

see (10) for an explicit one-loop example. Note that the frequency routing can be chosen separately for each loop in order to facilitate the computation. To sum up, the only necessary properties for a given analytic continuation of Euclidean correlation functions are (i) the equivalence on the Matsubara frequencies $\omega=2 \pi n T$, and (ii) the difference to the real time correlation function is accessible, see (5). Hence, as (i) is trivially satisfied, the 
relation (5) is at the heart of a numerical computation of the real time propagators, and in particular the spectral function.

Thus, the key step is the computation of $G(-\mathrm{i} \omega, \vec{p})$ at complex frequencies $\omega \in \mathbb{C}$ from the Euclidean propagator $G_{\mathrm{E}, \mathrm{cont}}(\omega, \vec{p})$. The latter is directly accessible in the present FRG framework. The difference is entailed in (5), and the pole positions and the corresponding residues can also be computed numerically.

The numerical FRG-computation of the propagator $G_{\mathrm{E}, \mathrm{cont}}(\omega, \vec{p})$ is facilitated by the fact, that all FRGrelations for correlation functions are one-loop exact. This is very amiable for computations at complex external frequency, as one only has to numerically follow poles and cuts at one loop. It also allows to discuss the important properties of such a procedure already at the perturbative one-loop example with classical propagators and vertices. The additional pole and cut structures arising from the introduction of momentum- and frequencydependent regulators are then discussed in a second step.

Hence, we first consider the one-loop correction to the Euclidean propagator $\Delta \Gamma^{(2)}$ that arises from a loop diagram with three-point vertices $\Gamma^{(3)}$. The latter are assumed to be momentum-independent, and we allow for two different propagators $G_{1}$ and $G_{2}$, corresponding to masses $m_{1}$ and $m_{2}$ respectively. This already encompasses the important case of loops with radial modes $\sigma$ and (pseudo-) Goldstone modes $\vec{\pi}$. In low-energy effective theories of QCD these stand for the lowest scalarpseudo-scalar meson multiplet, the sigma meson and the pions. The corresponding one-loop contribution for Euclidean external frequencies reads

$$
\begin{aligned}
\Delta \Gamma_{\mathrm{E}, \mathrm{cont}}^{(2)}(p) & =\left[\Gamma^{(3)}\right]^{2} \bigvee_{q} G_{1}(q) G_{2}(p+q) \\
& =\left[\Gamma^{(3)}\right]^{2} \bigvee_{q} \frac{1}{q_{0}^{2}+\left(\epsilon_{q}^{1}\right)^{2}} \frac{1}{\left(q_{0}+p_{0}\right)^{2}+\left(\epsilon_{q+p}^{2}\right)^{2}},
\end{aligned}
$$

with constant classical vertex $\Gamma^{(3)}$ and the quasiparticle energies

$$
\epsilon_{q}^{i}=\sqrt{\vec{q}^{2}+m_{i}^{2}} .
$$

In (6) and in the following we use the shorthand notations

$$
\int_{q} \equiv \int \frac{\mathrm{d}^{4} q}{(2 \pi)^{4}}, \quad \Varangle_{q} \equiv T \sum_{q_{0}} \int \frac{\mathrm{d}^{3} q}{(2 \pi)^{3}}, \quad \int_{\vec{q}} \equiv \int \frac{\mathrm{d}^{3} q}{(2 \pi)^{3}} .
$$

In (6) we have chosen the frequency routing in the diagram such that one of the propagators, $G_{1}$, only depends on the loop frequency $q_{0}=2 \pi n T$ with $n \in \mathbb{Z}$. For external Matsubara frequencies $p_{0}=2 \pi n T$ this agrees with the routing $G_{1}(q-p) G_{2}(q)$ and other routings that are obtained by shifts with Matsubara frequencies. For $p_{0} \neq 2 \pi n T$ all these choices are different. Moreover, the analytic continuation specified with the frequency routing in (6) does not coincide with (4) for $p_{0} \neq 2 \pi n T$ with $n \in \mathbb{Z}$. This also holds for any other frequency routing.

In the present perturbative one-loop example the above frequency routing is very ad hoc as there is not even a selection criterion for choosing $G_{1}(q)$ instead of $G_{2}(q)$. In the functional renormalisation group approach diagrams such as (6) are hit by derivatives w.r.t. the infrared cutoff scale that only act on the propagators, see (18). For diagrams with $n$ propagators this leads to $n$ diagrams instead of one, and singles out exactly one propagator in each of these diagrams. This provides the selection criterion which makes the above frequency routing uniquely defined. Importantly, this specific routing also simplifies the numerics.

Evidently (6) satisfies the first of the two properties of an analytic continuation defined below (5), it agrees with the Euclidean result for external Matsubara frequencies. As argued above, it is also uniquely defined, at least in the functional renormalisation group approach. This is an important prerequisite for the second property, access to the difference between the real time correlation functions and the specific analytic continuation of the Euclidean ones. This requires a discussion of the pole structure of (6). With the bosonic thermal distribution

$$
n\left(\omega_{\mathrm{M}}\right)=\frac{1}{e^{\beta \omega_{\mathrm{M}}}-1},
$$

for Minkowski frequencies we can rewrite (6) in terms of a contour integral surrounding the Euclidean axis. The Matsubara frequencies are then the positions of the poles at $\omega_{E}=2 \pi n T$ with $n \in \mathbb{Z}$. For Euclidean frequencies $p_{0} \in \mathbb{R}$ the integral is easily performed and we arrive at

$$
\begin{aligned}
& \Delta \Gamma_{\mathrm{E}, \mathrm{cont}}^{(2)}(p) \\
& =\frac{\left[\Gamma^{(3)}\right]^{2}}{2 \mathrm{i}} \sum_{ \pm} \int_{\vec{q}}\left(\underset{ \pm \mathrm{i} \epsilon_{q}^{1}}{\operatorname{Res}}\left[G_{1} G_{2}\right] \cdot\left[1+2 n\left(\mp \epsilon_{q}^{1}\right)\right]\right. \\
& \left.+\underset{-p_{0} \pm \mathrm{i} \epsilon_{q+p}^{2}}{\operatorname{Res}}\left[G_{1} G_{2}\right] \cdot\left[1+2 n\left(-\mathrm{i} p_{0} \mp \epsilon_{q+p}^{2}\right)\right]\right) .
\end{aligned}
$$

The expression $\operatorname{Res}_{r_{0}}\left[G_{1} G_{2}\right]$ in $(10)$ denotes the residue of the integrand $G_{1}(q) G_{2}(p+q)$ at $q_{0}=r_{0}$. Coming back to the discussion which lead to (5), to obtain the contribution one requires the proper analytic continuation of 10 to imaginary momenta, which is not uniquely defined at finite temperatures, where the propagator is only given at discrete Matsubara frequencies. Then, the additional requirement of appropriate analyticity conditions 21] singles out a unique continuation. This ambiguity is already visible in (10), where the omission of the external momentum in the argument of the second distribution function leads to a different continuation which coincides with $(10)$ for $p_{0} \in 2 \pi \mathbb{Z}$. The desired continuation to obtain the retarded correlation function can be determined within a real time formalism such as the closed time path 


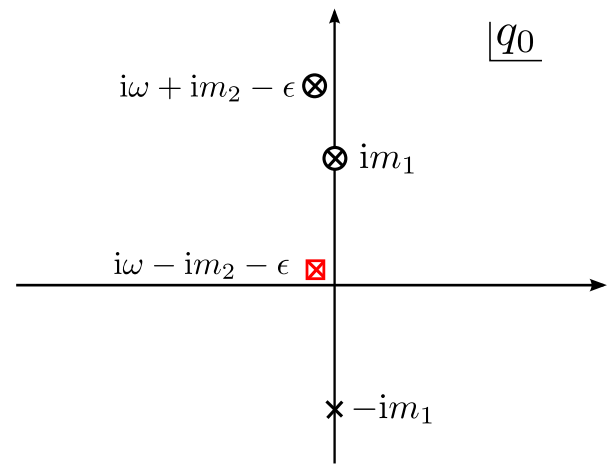

FIG. 1. Pole structure for the one-loop calculation at $T=0$.

formalism discussed below. Here the real time result is given by 10 omitting the external momentum in the argument of the distribution function. This result coincides with that obtained from the standard continuation procedure in the imaginary time formalism [22, 23], where the periodicity of the distribution functions for Euclidean external momenta is exploited before the analytic continuation is performed.

In the present example the difference between the two continuation procedures only consists of residues of poles and no cuts. Explicitly it is given by

$$
\begin{aligned}
& \Delta \Gamma_{\text {res }}^{(2)}(\omega, \vec{p} ; \epsilon) \equiv \Delta \Gamma_{\mathrm{E}, \text { cont }}^{(2)}(\omega, \vec{p} ; \epsilon)-\Delta \Gamma_{\mathrm{R}}^{(2)}(\omega, \vec{p} ; \epsilon) \\
= & \left.(-\mathrm{i})\left[\Gamma^{(3)}\right]^{2} \int_{\vec{q}} \sum_{ \pm} \operatorname{Res}_{-p_{0} \pm \mathrm{i} \epsilon_{q+p}^{2}} G_{1} G_{2}\right|_{p_{0} \rightarrow-\mathrm{i}(\omega+\mathrm{i} \epsilon)} \\
& \times\left(n\left(-\omega+\mathrm{i} \epsilon \mp \epsilon_{q+p}^{2}\right)-n\left(\mp \epsilon_{q+p}^{2}\right)\right) .
\end{aligned}
$$

In particular, for vanishing temperature, $T \rightarrow 0$, where $n(z) \rightarrow-\Theta(-\operatorname{Re} z)$, we have

$$
n\left(-\omega+\mathrm{i} \epsilon \mp \epsilon_{q+p}\right)-n\left(\mp \epsilon_{q+p}\right) \rightarrow \pm \Theta\left(\mp \omega-\epsilon_{q+p}\right) .
$$

This implies in particular that at vanishing temperature both results agree for small Minkowski external momenta $|\omega|<m_{2}$. This is simple to understand from the pole structure shown in Fig. 1. At zero temperature the difference between the two results is just given by the contribution of the circled pole in Fig. 1, which enters the upper/lower half plane for $|\omega|>m_{2}$. However, even at nonvanishing temperature the desired $\Gamma_{\mathrm{R}}^{(2)}(\omega, \vec{p})$ can be computed entirely numerically via

$$
\Gamma_{\mathrm{R}}^{(2)}(\omega, \vec{p})=-\lim _{\epsilon \rightarrow 0}\left(\Gamma_{\mathrm{E}, \mathrm{cont}}^{(2)}(\omega, \vec{p} ; \epsilon)-\Delta \Gamma_{\mathrm{res}}^{(2)}(\omega, \vec{p} ; \epsilon)\right) .
$$

The calculation of $\Gamma_{\mathrm{E} \text {,cont }}^{(2)}$ involves a straightforward Euclidean integration/ Matsubara sum. The difference $\Delta \Gamma_{\mathrm{res}}^{(2)}(\omega, \vec{p} ; \epsilon)$ is calculable given the residues of the integrand, that can be determined numerically. The advan- tage of such a procedure is that given appropriate regulator functions such as the ones discussed in the next section, the momentum integration/Matsubara summation can be carried out as before; it only remains to trace poles of the propagators and calculate corresponding residues in order to obtain the full real time result.

\section{B. General-purpose regulators for complex momenta}

The previous section gave us a very clear picture of how to treat the poles of the Euclidean propagator in order to obtain the retarded real time correlation function. In the present work we aim at evaluating spectral functions in the framework of the FRG. There, an infrared cutoff is introduced by means of a momentum- and/or frequencydependent modification of the classical kinetic term. For the simple example of a real scalar theory this entails

$$
\int_{p} \phi(p) p^{2} \phi(-p) \rightarrow \int_{p} \phi(p)\left(p^{2}+R_{k}\left(p_{0}, \vec{p}\right)\right) \phi(-p)
$$

For low spatial momenta/frequencies the cutoff function $R_{k}(p / k \rightarrow 0) \approx k^{2}$ acts as a mass term. In turn, $R_{k}(p / k \rightarrow \infty) \rightarrow 0$ ensures that the ultraviolet is unchanged. Lowering the infrared cutoff scale $k$ leads to a successive integration of the momentum and/or frequency fluctuations at about the cutoff scale. The scale dependence of the effective action $\Gamma_{k}[\phi]$ of the theory at hand is governed by the Wetterich equation [24],

$$
\partial_{t} \Gamma_{k}[\bar{\phi}]=\frac{1}{2} \operatorname{Tr} G[\bar{\phi}] \partial_{t} R_{k}, \quad t=\log k / \Lambda,
$$

with the propagator $G[\bar{\phi}]$ defined in $\sqrt{4}$ in the presence of a general background. In terms of the effective action it reads

$$
G[\bar{\phi}]=\frac{1}{\Gamma_{k}^{(2)}[\bar{\phi}]+R_{k}} .
$$

In 15 we have also introduced some reference scale $\Lambda$, usually being the UV scale, where the flow is initialised.

In the present work we shall discuss the case of general correlation functions at the example of the propagator. Its flow, or rather that of $\Gamma_{k}^{(2)}$ is derived from 15 by taking the second derivative w.r.t. $\phi$. The corresponding diagrams are directly linked to the one-loop diagrams in the last section. This is seen by rewriting (15) as

$$
\partial_{t} \Gamma_{k}[\bar{\phi}]=\left.\frac{1}{2} \operatorname{Tr} \partial_{t}\right|_{\Gamma_{k}^{(n)}} \ln \left(\Gamma_{k}^{(2)}[\bar{\phi}]+R_{k}\right) .
$$

$\phi$-derivatives commute with the partial $t$-derivatives and hence the flows of $n$-point functions take the form of partial $t$-derivatives of the corresponding one-loop diagrams with full propagators and vertices. The partial $t$-derivative only hits the $R_{k}$ dependence of the propagators with

$$
\left.\partial_{t}\right|_{\Gamma_{k}^{(n)}} G=\tilde{\partial}_{t} G=-G \dot{R}_{k} G, \quad \text { with } \quad \dot{R}_{k}=\partial_{t} R_{k},
$$


where we have introduced the notation $\tilde{\partial}_{t}$ for the partial $t$-derivatives at fixed $\Gamma_{k}^{(n)}$. The flow of correlation functions, $\partial_{t} \Gamma^{(n)}$, is derived from 15 by taking the $n$th derivative w.r.t. the fields. With

$$
\frac{\delta}{\delta \phi} G=-G \Gamma^{(3)} G, \quad \frac{\delta}{\delta \phi} \Gamma^{(m)}=\Gamma^{(m+1)},
$$

this leads to a sum of general one loop diagrams in full vertices and propagators for the flows $\partial_{t} \Gamma^{(n)}$. However, in each diagram one of the lines is given by $G \dot{R}_{k} G$. As already discussed in the last section, this allows us to define a unique analytic continuation procedure in frequency space as follows: In each diagram contributing to the flow of a given correlation function $\Gamma_{k}^{(n)}$ we choose the frequency routing such that $G \dot{R}_{k} G(q)$ has the Euclidean loop frequency $q_{0}$. This entails that we only have to discuss the poles of the propagator $G\left(q+\sum p_{i}\right)$ itself as the frequency argument of $G \dot{R}_{k} G(q)$ is Euclidean. It even only takes values on the Matsubara frequencies. Here $p_{i}$ are the external four-momenta with possibly complex frequencies $\left(p_{0}\right)_{i}$. Note that the general analysis also includes the poles of the vertices, the analysis of which will be published elsewhere.

Accordingly, the pole analysis of the propagator poles of the previous Section IIA carries over to the present case. However, the infrared regulator function changes the pole (and cut) structure discussed above in Section IIA:

First of all, the regulator necessarily triggers cutoffdependent shifts of the location of the poles evaluated in Section IIA. This also extends to possible cuts in the complex plane, see [11, 13 15] for an extensive discussion, that have not been discussed in Section IIA. However, if these $k$-dependent cuts are present for the one-loop diagrams in full propagators and vertices, the partial $t$ derivative converts them into poles. The cuts are reinstated within the $k$-integration due to the $k$-propagation of the poles. The propagation of the latter has to be followed anyway, and cuts pose no further problem. Secondly, in the case of frequency-dependent regulators further, unphysical poles are generated by the regulator itself.

In summary this leaves us with two options: We avoid the unphysical regulator poles with a regulator that only depends on spatial momenta. However, this comes at the price of a breaking Euclidean and Minkowski symmetry. The second option is to use space-time symmetrypreserving regulator functions, that are functions of the four-momentum squared. Then, the regulator poles cannot be avoided.

Here, we are particularly interested in the case of symmetry-preserving regulator functions in contradistinction to the predominantly used spatial regulator functions in first applications towards a direct computation of real time correlation functions. Such symmetrypreserving regulator functions are an indispensable prerequisite for studies of complex systems involving nontrivial momentum and frequency dependencies, see e.g.

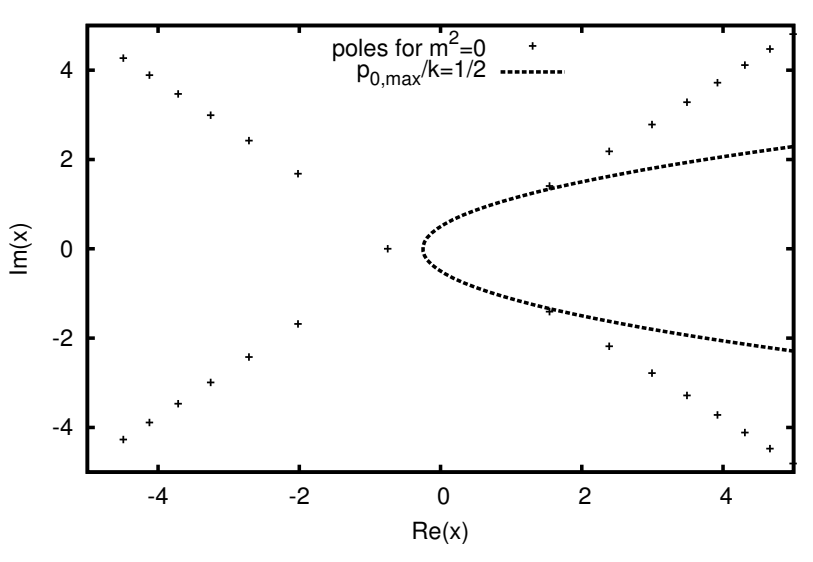

FIG. 2. Poles of the regularised propagator in the complex $x=\left(p_{0}^{2}+\vec{p}^{2}\right) / k^{2}$-plane for $m^{2}=0$ and a double exponential regulator. The parabola represents the boundary of accessible momenta for $p_{0, \max } / k=1 / 2$.

[3] for a possible application in QCD. Moreover, most approximation schemes in use within FRG applications are built on momentum locality: they do not take into account the full momentum dependence of all correlation functions at finite order of the expansion scheme. This applies in particular to the LPA-type expansion used here. Such expansions asks for regulators that minimise the momentum transfer in the flow diagrams, see [25].

Regulators that minimise the momentum transfer in the diagrams necessarily need a rapid decay with frequency and spatial momenta. Such a rapid decay is directly linked to the occurrence of a large number of unphysical poles, in the case of the numerically welltractable exponential decay one has to deal with a whole series of poles in the complex plane. In Fig. 2 we show the propagator 16 in the presence of a double exponential regulator,

$$
R_{k}\left(p^{2}\right)=p^{2} r\left(p^{2} / k^{2}\right), \quad r(x)=\frac{x}{e^{x^{2}}-1},
$$

and $\Gamma_{k}^{(2)} \rightarrow p^{2}$ for the sake of simplicity. Eq. 20 is a specific case of a general class of regulators with exponential decay introduced later, see (23). Fig. 2 shows an infinite number of additional regulator poles. Note that in the present massless case the poles remain fixed in the dimensionless $x=\left(p_{0}^{2}+\vec{p}^{2}\right) / k^{2}$-plane, for the general analysis see App. A3.

As additional regulator poles at finite $k$ turn out to be unavoidable, the following observation is helpful: The calculation of spectral functions $\rho(\omega, \vec{p})$ with external frequencies $|\omega|<p_{0, \max }$ utilises propagators in a strip $\mathcal{S}_{p_{0}, \max }$ with $\left|\operatorname{Im} q_{0}\right|<p_{0, \max }$ around the real axis in the complex $q_{0}$-plane as input. Therefore one requires regulator functions which vanish in the strip as $k \rightarrow 0$,

$$
\lim _{k \rightarrow 0} R_{k}(q+\mathrm{i} p) \rightarrow 0 \quad \text { for } q \in \mathbb{R} \text { and }\left|p_{0}\right|<p_{0, \max },
$$




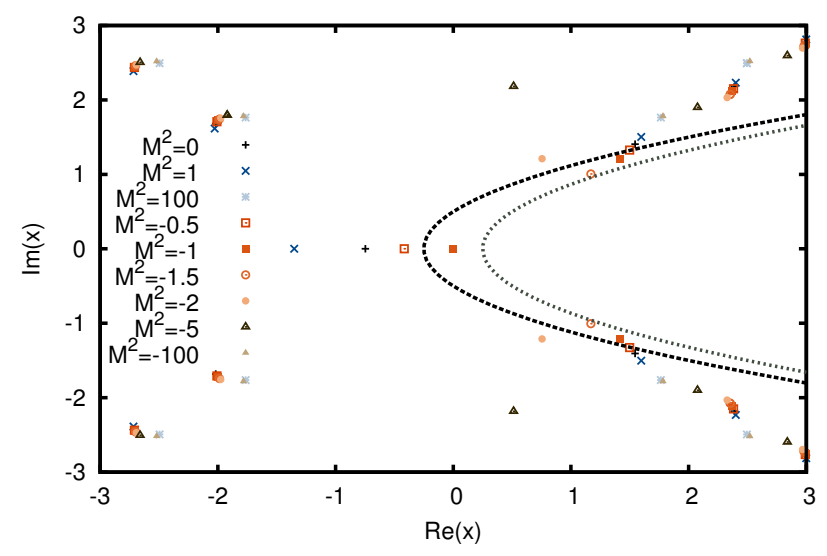

FIG. 3. Physical (on the negative real axis) and regulator poles in the complex $x=\left(p_{0}^{2}+\vec{p}^{2}\right) / k^{2}$ plane for different values of $M^{2}=\left(m^{2}-\Delta m_{r}^{2}\right) / k^{2}$, see App. A3 for details. The black(grey) parabola represents the boundary of $\mathcal{S}_{p_{0}, \max }$ for $\Delta m_{r} / k=0\left(\Delta m_{r} / k=1 / 2\right)$ for $p_{0, \max } / k=1 / 2$.

where here and in the following we use the shorthand notation $q+\mathrm{i} p$ for $\left(q_{0}+\mathrm{i} p_{0}, \vec{q}+\vec{p}\right)$. Note that the most desirable regulator function would preserve space-time symmetries but show at most physical poles at finite $\mathrm{RG}$ scales $k$. The restriction to external frequencies in $\mathcal{S}_{p_{0} \text {,max }}$ restricts the accessible momenta to a parabola in the complex $x=\left(p_{0}^{2}+\vec{p}^{2}\right) / k^{2}$-plane, see Fig. 2 .

The calculation of Minkowski correlation functions from the Euclidean ones requires the integration along deformed contours in the complex $q_{0}$ plane. Hence, on the one hand we need analytic regulator functions in order to be able to apply analyticity arguments. On the other hand we aim for regulator functions which at finite RG-scales $k$ introduce as few additional poles in the strip as possible.

Here, we construct regulator functions that do not lead to any additional regulator poles in addition to the physical poles in the strip $\mathcal{S}_{p_{0}, \max }$ at finite $k$. There are various ways of implementing these constraints, and we have evaluated many of them. Some of them, including the ensuing symmetry and analyticity constraints are discussed in App. A for the benefit of the reader.

Here we put forward a specific construction that has turned out to be the most flexible and stable one for our purposes. The central idea is to introduce an artificial mass term $\Delta m_{r}^{2}$ also in the regulator shape function $r$, which moves the additional regulator poles outside the strip. Explicitly, we only discuss the very convenient class of regulators that are also used in the subsequent numerical implementation,

$$
R_{k ; \Delta m_{r}^{2}}\left(p^{2}\right)=\left(\left.\Delta \Gamma_{k}^{(2)}\left(p^{2}\right)\right|_{\phi=\phi_{0}}+\Delta m_{r}^{2}\right) r\left(\frac{p^{2}+\Delta m_{r}^{2}}{k^{2}}\right),
$$

The regulator introduced in 22 is proportional to the momentum-dependent part $\Delta \Gamma_{k}^{(2)}\left(p^{2}\right)=\Gamma_{k}^{(2)}\left(p^{2}\right)-$

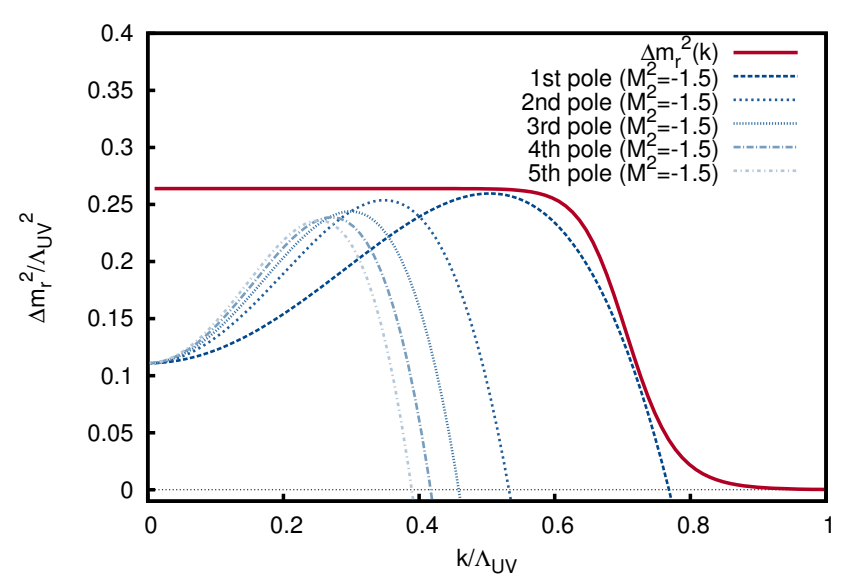

FIG. 4. Constraints on $\Delta m_{r}^{2}(k)$ from avoiding additional poles in the parabolic region as obtained from (A11) here for $p_{0, \max }=0.33 \Lambda_{\mathrm{UV}}$ and a double exponential regulator $(m=2)$. The solid red line shows our parameterisation $\Delta m_{r}^{2}(k)=\alpha\left|p_{0, \max }\right|^{2}\left(1+\left(\beta k /\left|p_{0, \max }\right|\right)^{n}\right)^{-1}$ for $\alpha=2.38$, $\beta=0.47$ and $n=20$, see App. A 3 for details.

$\Gamma_{k}^{(2)}(0)$ of the inverse propagator. We want to stress at this point that the described procedure to avoid artificial regulator poles is of general nature and can be applied not only to regulators of the form $(22)$ with general shape functions but to arbitrary regulators that work for real momenta.

Fig. 3 shows the pole structure of the propagator in the presence of a finite $\Delta m_{r}$-parameter and different mass parameters $m^{2}$, see App. A3 for a detailed discussion. The main effect of the latter is to shift the parabolic region of accessible momenta to the right whereas the location of the poles is only shifted slightly. This analysis implies that it is always possible to avoid regulator poles in $\mathcal{S}_{p_{0}, \max }$ by choosing an appropriately large $\Delta m_{r}^{2}$ at every scale. This implies in particular that one can start with a vanishing $\Delta m_{r}^{2}$ in the UV for $k \rightarrow \Lambda_{\mathrm{UV}}$ with $\Lambda_{\mathrm{UV}} \gg$ physical scales, where the regulator poles are still far outside the strip.

For our explicit computations we further restrict 22 . to a general class of exponential decay regulators with

$$
r(x)=\frac{x^{m-1}}{e^{x^{m}}-1} .
$$

Fig. 4 shows the constraints arising from avoiding the first few propagator poles in the complex plane for the regulator with $m=2$ in 23 in units of $\Lambda_{\mathrm{UV}}$. These poles can obviously be avoided by a $k$ dependence of $\Delta m_{r}^{2}$ in the form of a smooth theta function with appropriately chosen parameters, see App. A3 for details.

The inclusion of $\Delta m_{r}^{2}$ leads to a substantial modification of the regulator's effective cutoff scale [25, 26] compared to the RG-scale $k$. In particular there is an exponential drop of the physical cutoff scale $k_{\text {eff }}$ with $\Delta m_{r}^{2}$ in the regime $k^{2} \lesssim \Delta m_{r}^{2}$, see Fig. 5 and App. A 5 for a de- 


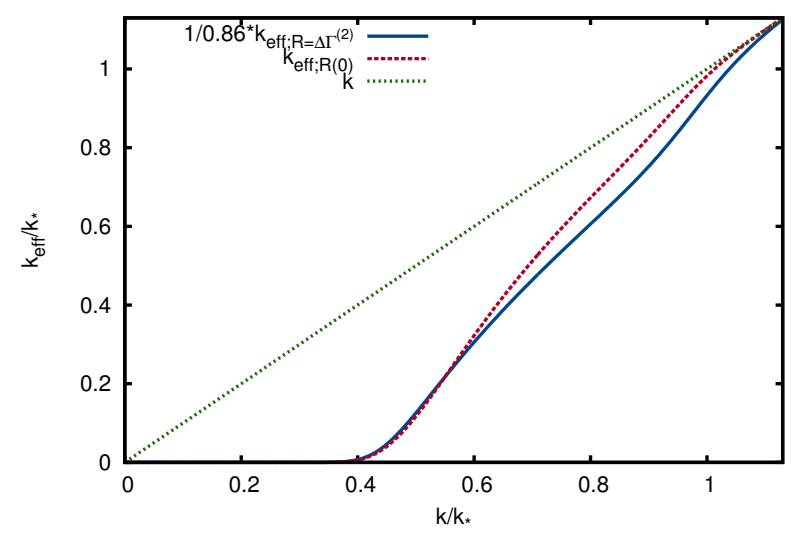

FIG. 5. Effective cutoff scales $k_{\text {eff; } R=\Delta \Gamma^{(2)}}$ and $k_{\text {eff; } R(0)}$ as a function of $k / k_{*}$, where $\Delta m_{r}^{2}\left(k_{*}\right) / \Delta m_{r}^{2}(0)=0.5$, here for the same parameter set as in Fig. 4

tailed discussion. We conclude $\Delta m_{r}^{2}(k)$ should be chosen as small as possible for several reasons.

Firstly we would like to keep the comparability to the standard regulator case with $\Delta m_{r}^{2}=0$, hence allowing for direct access to the plethora of results obtained there. While this merely is convenient, we encounter a more severe constraint in theories with several different field modes with different mass scales. There, the exponential drop of the physical cutoff scales $k_{\text {eff }}$ relative to the cutoff parameter $k$ causes the momentum fluctuations of the respective fields to quickly disentangle in the regime $\Delta m_{r}^{2} \lesssim k^{2}$ for at least one of the modes. In other words, in this regime, the flow integrates out momentum and frequency modes of the different fields at potentially vastly different momentum scales. This either asks for approximation schemes that are amiable for a large momentum transfer or a very accurate determination of the relative physical cutoff scales $k_{\text {eff }}$.

In principle, both properties can be adjusted for. For example, a vertex expansion keeping the full momentum dependence of the correlation functions has been put forward in 3 for QCD. However, the computational effort is relatively large and we aim at an approach working for general approximation schemes. Appropriately defined effective cutoff scales can be used to adjust relative cutoff scales to keep the momentum transfer small in the first place. This allows using simpler truncations without considering the full momentum dependence.

The accurate determination of the relative physical cutoff scale is the subject of App. A5 and of ongoing work. In any case, the problem of a potential momentum transfer is minimised by minimising $\Delta m_{r}^{2}$. Therefore, one should for example refrain from using a divergent $\Delta m_{r}^{2}(k)$ as $k \rightarrow 0$, that would in principle allow for arbitrarily large Minkowski frequencies.

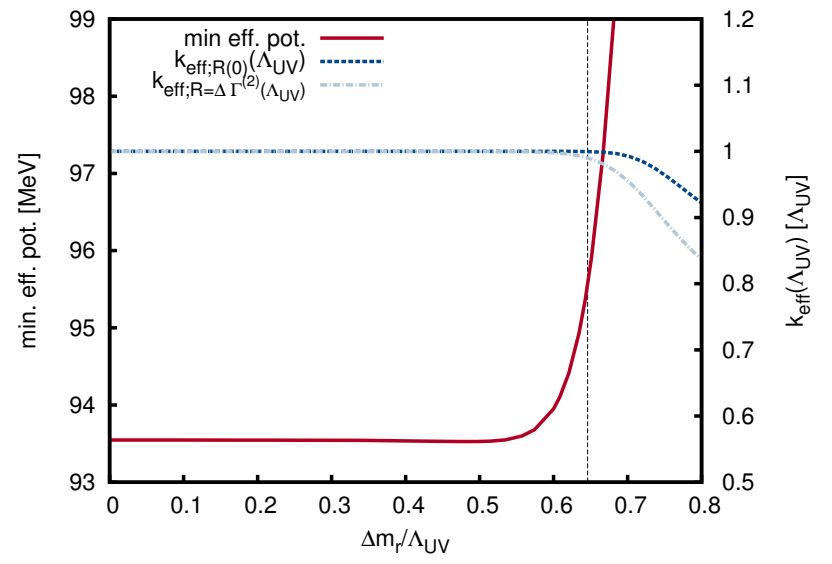

FIG. 6. Minimum of the effective potential and effective cutoff scales $k_{\text {eff }}\left(\Lambda_{\mathrm{UV}}\right)$ in dependence of $\Delta m_{r}$ obtained by varying $p_{0, \max }$ for fixed parameter values $\alpha=2.38, \beta=0.47$ and $n=20$ in $\mathrm{A} 6$. The dashed vertical line denotes the value for $\Delta m_{r} / \Lambda_{\mathrm{UV}}$, where $k_{\mathrm{eff} ; R=\Delta \Gamma^{(2)}}\left(\Lambda_{\mathrm{UV}}\right)$ deviates more than $1 \%$ from $\Lambda_{\mathrm{UV}}$. For comparison, the value $p_{0, \max }=300 \mathrm{MeV}$ used in the calculation of the spectral functions corresponds to a value $\Delta m_{r} / \Lambda_{\mathrm{UV}} \approx 0.51$.

\section{Application to the $O(N)$ model}

The approach to FRG computations of correlation functions at complex frequencies can now readily be applied to general theories including fermionic fields. No further conceptual or technical problems have to be solved. Note also that the current set-up is also applicable at finite chemical potential. In a Euclidean approach the latter technically is nothing but an imaginary frequency in all correlation functions. Applications to single spectral functions in QCD and low-energy effective models of QCD at vanishing and finite temperature and density are under way. Apart from providing interesting physics information about the decay and formation of resonances, they serve as input in the direct real time computation of transport coefficients within the approach put forward in [1, 2], see also [27 30] for recent effective model calculations. Respective results will be presented elsewhere.

Here we illustrate the power of the present approach for numerical computations with the calculation of spectral functions in the $O(N)$ model at vanishing temperature in the local potential approximation (LPA). The $O(N)$ model is a simple model for the mesonic low-energy dynamics. It is well-known that, despite its formal simplicity, the solution of the $O(N)$ model in LPA is numerically far more involved than related quark-meson or NJL-type models in LPA-type approximations. This is related to the dominance of fermionic short-range fluctuations in the latter class of models, as compared to the dominance of pion long-range fluctuations in the former. Note that in advanced approximations beyond LPA all models have to face the numerical subtleties mentioned above. It is 


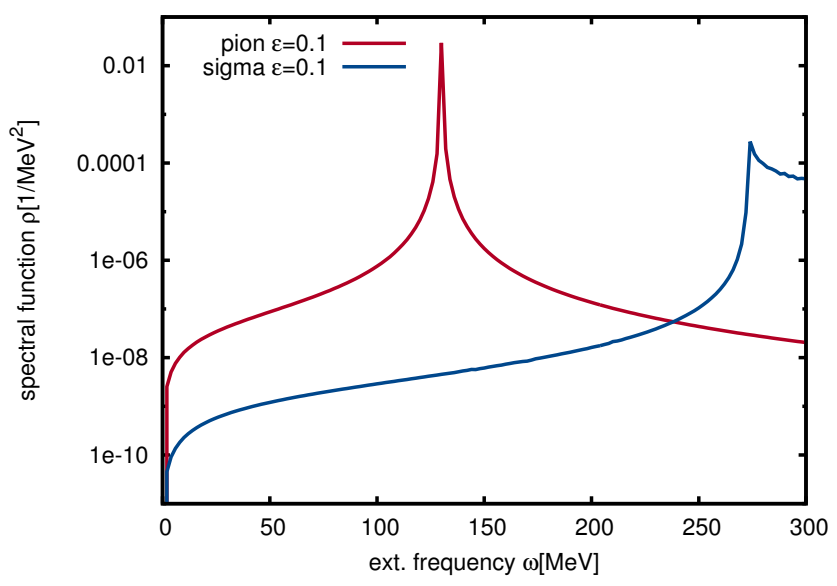

FIG. 7. Spectral functions of the $O(N)$ model at vanishing temperature using a $4 \mathrm{~d}$ regulator function for $\epsilon=0.1 \mathrm{MeV}$.

this fact which makes the $O(N)$-model in LPA the ideal test case.

In LPA we take into account the full, scale dependence in the effective potential, the effective action evaluated at constant background fields, divided by the fourdimensional volume $\mathcal{V}$,

$$
V_{k}(\phi)=\frac{1}{\mathcal{V}} \Gamma_{k}[\phi], \quad \text { with } \quad \mathcal{V}=\int \mathrm{d}^{4} x .
$$

In the LPA, the effective potential is computed from its flow with the Ansatz

$$
\Gamma_{k}[\phi]=\int_{x}\left[\frac{1}{2}\left(\partial_{x} \phi_{i}\right)^{2}+V_{k}\left(\phi^{2}\right)\right],
$$

which gives a closed flow equation for $V_{k}$. Then the momentum- and complex-frequency-dependent flow of the propagator is computed with the vertices $V_{k}^{(3)}, V_{k}^{(4)}$ taken from the scale-dependent effective potential in the LPA approximation, see App. A4. This can be seen as the first iterative step in the computation of the fully selfconsistent computation of effective potential and propagator as put forward in 31. It has been shown there that the procedure converges rapidly and that in particular the momentum and frequency dependence of the propagator is already well approximated by the first iteration. The implementation follows App. A4 and represents a generalisation of $[8]$ to the case of symmetrypreserving $4 \mathrm{~d}$ regulator functions. Here we employ a Taylor expansion at a fixed expansion point 32 at a UV cutoff scale $\Lambda_{\mathrm{UV}}=900 \mathrm{MeV}$ using an exponential regulator with $m=2$ with parameters $\alpha=2.38, \beta=0.47$ and $n=20$ in Eq. A7) and map out the pion and the sigma meson spectral functions up to external real frequencies $\omega \leq p_{0, \max }=300 \mathrm{MeV}$, keeping a small but finite imaginary frequency $\epsilon=0.1 \mathrm{MeV}$. The UV parameters were tuned to yield physical parameters $f_{\pi}=93.6$ $\mathrm{MeV}, m_{\pi}=137 \mathrm{MeV}$ and $m_{\sigma}=425 \mathrm{MeV}$ in the IR.
As a first step we demonstrate the independence of the results of the chosen maximal value of $\Delta m_{r}^{2}$. This is illustrated in Fig. 6, where we show the impact of increasing $\Delta m_{r}^{2}$ on the minimum of the effective potential, which was tuned to physical values for a standard regulator with $\Delta m_{r}^{2}=0$. Here the crucial question is in how far the UV propagator is modified by the $\Delta m_{r}^{2}$-regulator compared to the standard regulator. This can be quantified by considering effective initial cutoff scales $\Lambda_{\text {eff }}=k_{\text {eff }}\left(\Lambda_{\mathrm{UV}}\right)$ as a function $\Delta m_{r} / \Lambda_{\mathrm{UV}}$. As soon as $\Lambda_{\text {eff }}$ starts to deviate significantly from $\Lambda_{\mathrm{UV}}$ the results at $k=0$ are changed. As shown in Fig. 6 for $\Delta m_{r} / \Lambda_{\mathrm{UV}} \gtrsim 0.65$ this deviation exceeds $1 \%$. The larger deviations in the minimum of the effective potential beyond this value can be understood on the level of the effective cutoff scale. For $\Delta m_{r} / \Lambda_{\mathrm{UV}} \gtrsim 0.65$ the effective initial scale $\Lambda_{\text {eff }}$ is lowered. In other words, then we initialise the flow at an effective lower scale $\Lambda_{\mathrm{eff}}<\Lambda_{\mathrm{UV}}$ with the same amount of symmetry breaking as initialised at $\Lambda_{\mathrm{UV}}$ for the reference flow at $m_{r}=0$. On the other hand, the effective cutoff scale does not capture all effects of $\Delta m_{r}^{2}$ on the momentumdependent propagator at the UV scale. For example at $\Delta m_{r} / \Lambda_{\mathrm{UV}}=0.61$, the respective propagator deviates by $1 \%$ from its counterpart at $\Delta m_{r}^{2}=0$ in the intermediate momentum regime, whereas the effective initial scales differ only by $0.3 \%$.

The expansion of the effective potential about the IRminimum of the effective potential similar to the expansion on a grid in field space, allows a direct computation of the two-point function at the minimum of the potential where exactly these couplings enter, which avoids having to expand both the potential and the two-point function about a scale-dependent minimum 8 . The flow equations for the two-point functions are then subsequently solved applying Eq. (13) by tracking the physical poles of the propagators in every $k$-step. The resulting spectral function is shown as a function of frequency in Fig. 7 for frequencies $\omega+i \epsilon$ and $\epsilon=0.1 \mathrm{MeV}$. The clear peak in the pion spectral function singles out the pole mass of the pion $m_{\pi, \mathrm{pol}}$, while the sigma shows the threshold of the $\sigma \rightarrow \pi \pi$ decay. Both structures get sharp in the limit $\epsilon \rightarrow 0$, more details and a quantitative comparison to earlier results obtained using a spatial flat regulator function [8] are provided in Sec. IIIB

In Fig. 7 the spectral functions are evaluated at vanishing spatial momentum $p=|\vec{p}|=0$. In our fully numerical procedure the extension to finite external spatial momentum represents only a minor complication as it only requires to evaluate an additional angular integration. The additional numerical costs are negligible, and the resulting spectral functions are shown in Fig. 8. For the pion spectral function the ridge follows the mass shell relation $\omega^{2}=m_{\pi, \mathrm{pol}}^{2}+\vec{p}^{2}$, while the $\sigma \rightarrow \pi \pi$ threshold follows the relation $\omega^{2}=4 m_{\pi}^{2}+\vec{p}^{2}$. The appearance of the curvature mass $m_{\pi}$ in the threshold relates to the missing completion of the iteration defined in [31] that yields the full frequency and momentum dependence of the propagators. In the fully iterated result we have $m_{\pi}^{2} \rightarrow m_{\pi, \mathrm{pol}}^{2}$. 


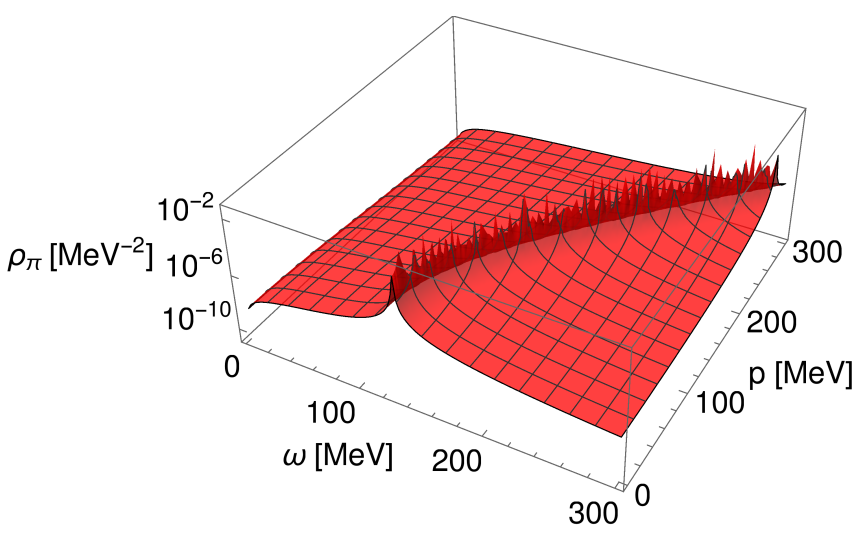

(a) Pion spectral function.

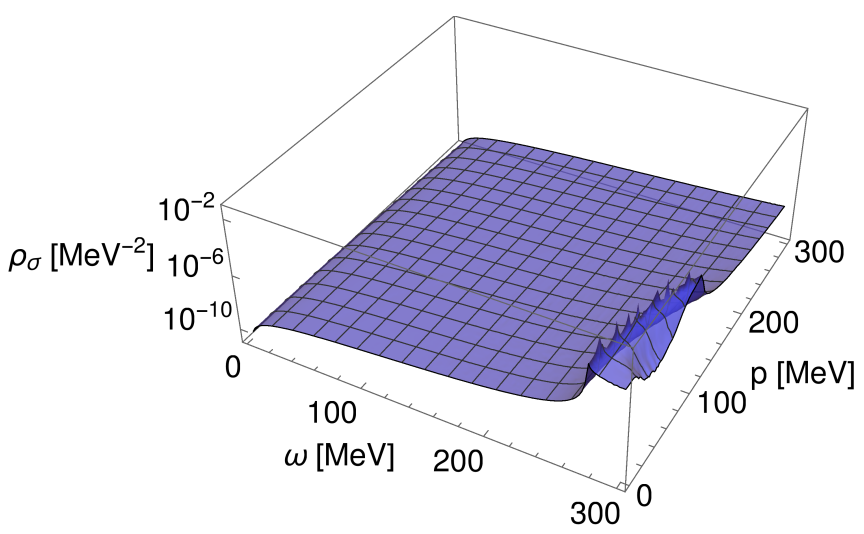

(b) Sigma spectral function.

FIG. 8. Mesonic spectral functions at vanishing temperature as a function of external frequency $\omega$ and momentum $|\vec{p}|$ obtained using a $4 \mathrm{~d}$ regulator function for $\epsilon=0.1 \mathrm{MeV}$.

For similar results within the quark-meson model and a spatial regulator function see [10].

We emphasise that $4 \mathrm{~d}$ regulators are particularly beneficial for imaginary time computations with full frequency and momentum dependencies at finite temperature, see [33, 34]. To see this it is illustrative to consider standard one loop Matsubara sums. These can be performed analytically and give rise to the well-known thermal suppression factors $\exp \left\{-m_{\text {gap }}(p) /(2 T)\right\}$ in the presence of a mass gap $m_{\text {gap }}(p)$. This has the additional benefit of a further UV regularisation of the spatial momentum integrations. In turn, a numerical computation of the corresponding Matsubara sums and spatial momentum integrals faces a combined numerical accuracy problem. Firstly, the exponential decay may involve large explicit sums of Matsubara frequencies before the spatial integration is performed. Reversing the order of numerical sum and integration leads to the requirement of an exponential accuracy of the momentum integration. In either way the numerical costs rise drastically in comparison to the vanishing temperature case. This applies in particular to the interesting transition region of $m_{\text {gap }}(p) \approx T$.

With $4 \mathrm{~d}$ regulators with rapid decay the above finite temperature problem is cured, and we only have to explicitly sum over a relatively small number of Matsubara frequencies. Related results on finite temperature spectral functions will be presented elsewhere. This is particularly relevant when considering the high accuracy requirements to obtain the imaginary part of the propagator when using a spatial regulator, which leads to significantly larger numerical costs.

\section{SPECTRAL FUNCTION FROM THE REAL TIME FORMALISM}

As a complementary second approach we discuss the direct calculation of spectral functions in a real time formalism [22, 23, 35, 36]. Real time applications of the
Functional RG were considered e.g. in $20,37,52$, for a recent review see also 53. Recent practical applications use spatial regulator functions [48, 52. Here we present the formalism required for the application of the real time formalism to the calculation of spectral function as starting point for future studies. This is not only interesting for the equilibrium case discussed here but, as mentioned in the introduction, also the appropriate formalism for applications in nonequilibrium [53, 54.

In order to preserve Lorentz invariance one would like to consider regulator functions which just depend on the Lorentz scalar $p^{2}=-p_{0}^{2}+\vec{p}^{2}$. In this case the integrand is just a function of $p^{2}$ and we can rewrite the momentum integration as a three-dimensional integration perpendicular to the mass-shell which is regularised and a one-dimensional integration along the mass-shell which is not regularised as the Lorentz-invariant regulator functions remain constant there. In order to achieve a regularisation on the mass-shell one is lead to consider regulator functions that break Lorentz invariance, to wit

$$
R_{k}\left(p_{0}, \vec{p}\right)=R_{k}\left(p^{2}\right)\left(r_{0}\left(p_{0}^{2} / k^{2}\right)+r_{s}\left(\vec{p}^{2} / k^{2}\right)\right) .
$$

The regulator in (26) is proportional to a Lorentzinvariant part $R_{k}\left(p^{2}\right)$, which provides a regularisation of off-shell fluctuations. A further factor is an energy or spatial momentum cutoff function which provides a regularisation on the mass shell. Alternatively one can employ just a Lorentz-invariant regulator $R_{k}\left(p^{2}\right)$ but then one has to carry out principle value integrations on the mass shell which requires knowledge about the position of the poles. Moreover, an accurate treatment of poles close to the Minkowski axis is required. This will be discussed elsewhere.

Regulators that also regularise frequencies and allow for straightforward real time computations, in the sense of no poles close to the real frequency axis, are necessarily complex, for example

$$
R_{k}\left(p_{0}, \vec{p} ; a\right)=(1+\mathrm{i} a) R_{k}\left(p_{0}, \vec{p}\right) .
$$




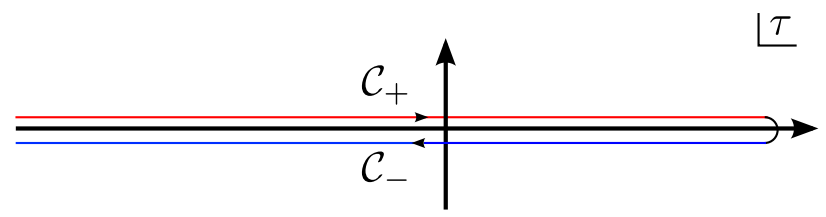

FIG. 9. Keldysh contour with forward and backward directions. The fields $\phi_{+}$live on the forward part of the contour $\mathcal{C}_{+}$, the fields $\phi_{-}$live on the backward part $\mathcal{C}_{-}$.

The regulators in (27) have the additional advantage that for $a>0$ and $R_{k}\left(p_{0}, \vec{p}\right)>0, \forall p_{0}, \vec{p}$ they automatically define Feynman propagators. Such regulators have a very clear physical interpretation as they introduce a finite width for all propagators. However, they lead to complex flows and introduce the necessity of an (additional) finetuning in order to remove unwanted complex parts of the effective action at $k=0$. Note also that they necessarily introduce additional poles that do not permit for simple analytic continuations of imaginary time results.

As a specific and simplest example we will focus in the following on applications involving purely spatial and in particular spatial flat regulator functions. In the classification from Eq. (26) spatial regulator functions correspond to the choice $R_{k}\left(p^{2}\right) \equiv k^{2}, r_{0} \equiv 0$ and $r_{s}(x)=\operatorname{xr}(x)$ for some generic shape function $r$. Such regulators also do not introduce additional poles to the complex plane of real and imaginary frequencies, and hence allow for a straightforward analytic continuation. For the calculation of spectral functions in the LPA, the real time procedure can be shown to be equivalent to the imaginary time procedure, but leads to a particularly convenient representation for numerical applications. With regard to the inclusion of general frequency dependencies the real time formalism is however the more flexible choice.

\section{A. Closed time path FRG-approach}

The closed time path (CTP) approach is based on the formulation of the theory on the Keldysh contour, see Fig. 9. For a scalar $O(N)$-theory, the field content is then doubled in $\phi_{+}$and $\phi_{-}$, living on the forward, $\mathcal{C}_{+}$, and backward part, $\mathcal{C}_{-}$, of the contour respectively. The action reads

$$
S\left[\phi_{+}, \phi_{-}\right]=S\left[\phi_{+}\right]-S\left[\phi_{-}\right], \quad S\left[\phi_{ \pm}\right]=\int_{\mathcal{C}_{ \pm}} \mathcal{L}\left(\phi_{ \pm}\right),
$$

where $x_{ \pm}$live on the forward/backward time contour respectively, $\int_{\mathcal{C}_{ \pm}}=\int d^{4} x_{ \pm}$, and the relative minus sign accounts for the direction of $x_{ \pm}$on the contour shown in Fig. 9. Naturally, also the regulator term has two parts, regularising the $\phi_{+}$and $\phi_{-}$terms,

$$
\frac{\mathrm{i}}{2} \int_{x_{+}} \phi_{+} R_{k}^{+} \phi_{+}+\frac{\mathrm{i}}{2} \int_{x_{-}} \phi_{-} R_{k}^{-} \phi_{-} .
$$

Eq. 29 can be generalised to regulators with mixed terms connecting $\phi_{+}$and $\phi_{-}$. Though potentially advantageous for technical reasons, such a procedure potentially spoils causality. The CTP flow equation then takes the form [20, 40, 42, 53 ]

$$
\partial_{t} \Gamma_{k}\left[\phi_{+}, \phi_{-}\right]=\frac{\mathrm{i}}{2} \operatorname{Tr} G_{++} \partial_{t} R_{k}^{+}+\frac{\mathrm{i}}{2} \operatorname{Tr} G_{--} \partial_{t} R_{k}^{-},
$$

where $\mathrm{i} G_{++}$and $\mathrm{i} G_{--}$are the ++ and -- components of the propagator. In thermal equilibrium the propagators can be parameterised solely in terms of the spectral function, see App. B1 and App. B2 for details.

In order to make use of the plethora of explicit resummations and structural results in perturbative thermal field theory, it is advantageous to rewrite the flow equation in terms of partial $t$-derivatives at fixed $\Gamma_{k}^{(n)}$ of the corresponding one loop diagrams (with full propagators and vertices) as already done in Section II B for the imaginary time formulation.

Moreover, the flow equation includes the flow of both parts of the contour. Hence it vanishes identically for $\phi_{+}=\phi_{-}=\phi, R_{k}^{+}=-R_{k}^{-}$, and \pm -symmetric initial conditions $\Gamma_{\Lambda}\left[\phi_{+}, \phi_{-}\right]=-\Gamma_{\Lambda}\left[\phi_{-}, \phi_{+}\right]$. A specific subclass of \pm -symmetric initial conditions is given by

$$
\Gamma_{\Lambda}\left[\phi_{+}, \phi_{-}\right]=\Gamma_{\Lambda}\left[\phi_{+}\right]-\Gamma_{\Lambda}\left[\phi_{-}\right],
$$

and includes the classical action 28). Taking a $\phi_{+^{-}}$or $\phi_{-}$-derivative breaks the \pm -symmetry and gives access to all correlation functions. This procedure yields for a $\phi_{+}$-derivative

$$
\partial_{\phi^{+}} \partial_{t} \Gamma_{k}=\frac{\mathrm{i}}{2} \tilde{\partial}_{t} \operatorname{Tr}\left(G_{a b} \Gamma_{b a+}^{(3)}\right),
$$

for the canonical choice $R_{k}^{+}=-R_{k}^{-}=R_{k}$ and $a, b= \pm$. The $t$-derivative at fixed $\Gamma_{k}^{(n)}, \tilde{\partial}_{t}$, only hits the propagator, see (18). For the LPA-based computation of spectral functions as discussed in the previous Section IIC we have to solve the flow equation for the effective potential. In analogy to 24 it is defined as

$$
V_{k}\left(\phi_{+}, \phi_{-}\right)=\frac{1}{\mathcal{V}} \Gamma_{k}\left[\phi_{+}, \phi_{-}\right],
$$

with the space-time volume $\mathcal{V}$. The LPA approximation at imaginary time 25 trivially extends to the CPTformulation at real time as

$$
\Gamma_{k}[\phi]=\sum_{ \pm}( \pm) \int_{x_{ \pm}}\left[\frac{1}{2}\left(\partial_{x} \phi_{ \pm}\right)^{2}+V_{k}\left(\phi_{ \pm}\right)\right],
$$

with the single field effective potential $V_{k}(\phi)$ with $V_{k}\left(\phi_{+}, \phi_{-}\right)=V_{k}\left(\phi_{+}\right)-V_{k}\left(\phi_{-}\right)$. Then, the flow equa- 
tion for the effective potential $V_{k}(\phi)$ reads,

$$
\begin{aligned}
\partial_{\phi} \dot{V}_{k}(\phi) & =\left.\partial_{\phi_{+}} \dot{V}_{k}\left(\phi_{+}, \phi_{-}\right)\right|_{\phi_{ \pm}=\phi} \\
& =-\frac{1}{2} \tilde{\partial}_{t} \operatorname{Tr}\left(\operatorname{Im} G_{++} \Gamma_{+++}^{(3)}\right) .
\end{aligned}
$$

In the LPA the spectral function is given by a delta function. In the simplest case of a spatial regulator function, i.e. for $R_{k}\left(p^{2}\right) \equiv k^{2}, r_{0} \equiv 0$ and $r_{s}(x)=x r(x)$ in 26 , which we will consider in the following, the frequency integration can be trivially performed. As expected, the flow equation for a spatial regulator function in the CTP formalism coincides with the flow equation for the effective potential derived in the imaginary time formalism, see App. B 3 for details.

To determine the spectral function or correspondingly real and imaginary parts of the retarded propagator, it is sufficient to derive flow equations for particular components, $\operatorname{Re} \Gamma_{++}^{(2)}$ and $\Gamma_{+-}^{(2)}$, of the inverse propagator, see App. B 1 for details. Again we consider the $O(N)$ model as simplest application where the flow equations for the real and imaginary parts of the retarded two-point functions are given explicitly by (B34) together with (B4). For the case of momentum-independent vertices, these equations are formulated in terms of $\tilde{t}$-derivatives of the loop integrals

$$
\begin{aligned}
\bar{J}_{j i}^{\mathrm{Re}}(p)= & \frac{1}{2} \int_{q} \operatorname{Im} G_{++}^{j}(q+p) \operatorname{Re} G_{++}^{i}(q) \\
& +\frac{1}{2} \int_{q} \operatorname{Re} G_{++}^{j}(q+p) \operatorname{Im} G_{++}^{i}(q) \\
\bar{J}_{j i}^{\operatorname{Im}}(p)= & -\frac{1}{2} \frac{\operatorname{sign}\left(p^{0}\right)}{2 n\left(p_{0}\right)} \int_{q} G_{+-}^{j}(q+p) G_{-+}^{i}(q) .
\end{aligned}
$$

In the LPA approximation, i.e. inserting delta functions for the spectral functions in the involved propagators, these take the form of the well-known one-loop expressions in the real time formalism [23] and are given explicitly in (B31) and (B33).

As discussed in detail in App. B5 the simplest way towards deriving explicit flow equations for a spatial flat regulator function is to insert the regulator at this point and to evaluate the $\tilde{t}$-derivative afterwards by exploiting the fact that it only acts on the explicit $k$ dependence in $\epsilon_{q}^{i}$ but not on mass term. In this way one derives explicit flow equations, which have a particularly simple structure for the imaginary part, see (B39). The corresponding flow equation shows delta functions in $k$. Therefore no numerical integration has to be carried out to evaluate the flow, see App. B5, which is particularly convenient for numerical purposes.

Despite its different appearance the flow equations for the retarded correlation functions can be shown to be formally equivalent to those derived starting from an imaginary time formalism [6 10, see App. B6. This reflects nothing but the equivalence of real time and imaginary time formalisms for the specific example of the evaluation of one-loop diagrams [23, 55]. Here the real time formalism lead to a representation where the $\epsilon \rightarrow 0$ limit in the transition from the Euclidean two-point function evaluated at complex external momentum and the retarded two-point function, see (2), is already taken. Such a formulation avoids high numerical costs for the evaluation of the flow equation in particular at finite temperature and density, see the discussion from above. From our point of view the real time approach is the preferable formalism to use for applications with spatial regulator functions, in particular with regard to the inclusion of further frequency dependencies in the flow.

We close with the remark that the above strategy can also be applied to covariant regulators. They have the advantage of full Lorentz invariance but additional care has to be taken concerning regulator poles close to the Minkowski axis as well as the numerical investigation of principle value integrals. While the former problem is resolved within the class of regulators defined in Sec. II B, the practical implementation of the latter requires special attention and will be discussed elsewhere.

\section{B. Application to the $O(N)$ model}

Fig. 10 shows the numerical result obtained by integrating the flow equations B34 using a set-up similar to the one described in App. A 4 . To ensure comparability of both results the UV parameters were chosen to yield the same physical parameters in the IR as in Sec. IIC, In particular we compare the result from keeping a finite imaginary part in the external momentum, implemented in the same way as in Sec. IIC and in the original works [8. 9], to the real time result where the limit $\epsilon \rightarrow 0$ has already implicitly been taken. As clearly visible from the $\sigma$-meson spectral function at large momenta both results agree once a genuine imaginary part in the retarded twopoint function builds up. The finite imaginary external momentum $\epsilon$ leads to an artificial broadening of the spectral functions below the thresholds where the imaginary part has to vanish identically at zero temperature. In this case the calculations can even be corrected by hand in order to obtain the full result with $\epsilon$ extrapolated to zero. The pion pole mass corresponding to the peak in the pion spectral function agrees in all cases as it is determined by the zero of the real part of the inverse retarded correlator which depends only weakly on $\epsilon$.

Coming to a comparison of the spatial regulator results from this section to the calculation using a $4 \mathrm{~d}$ regulator from Sec. II C, the most meaningful comparison comparison is that of two calculations with a fixed imaginary external frequency $\epsilon=0.1 \mathrm{MeV}$ keeping in mind the effects of the extrapolation $\epsilon \rightarrow 0$ as discussed above. Fig. 11 shows a striking agreement between the spectral functions from both calculations which is a highly nontrivial statement. In both cases the pion pole mass is given by $m_{\pi}^{\text {pole }}=131 \mathrm{MeV}$ compared to a curvature 


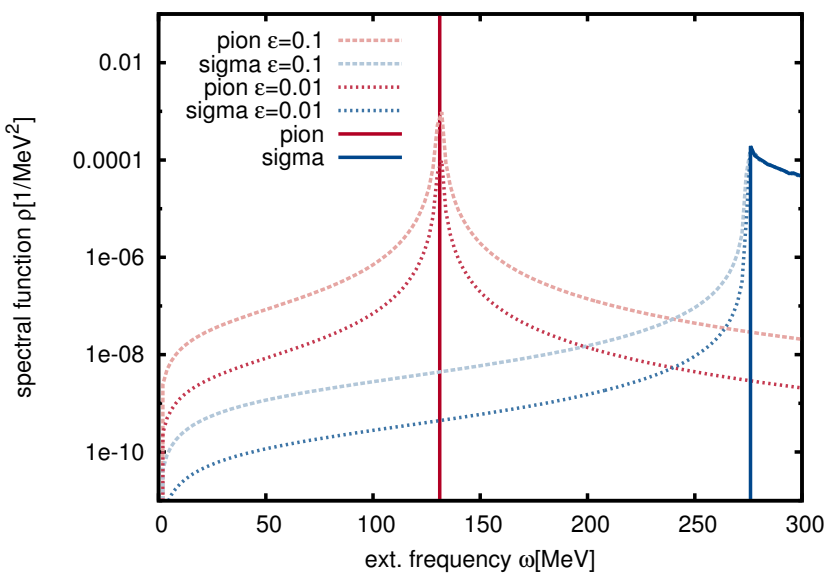

FIG. 10. Spectral functions of the $O(N)$ model at vanishing temperature comparing the result from the real time formalism to an implementation keeping finite imaginary parts $\epsilon=0.1 \mathrm{MeV}$ and $\epsilon=0.01 \mathrm{MeV}$ in the external momentum.

mass of $m_{\pi}=137 \mathrm{MeV}$. This difference of $5 \%$ in the LPA was shown to decrease with the inclusion of the full momentum dependence of the propagators, see 31.

\section{SUMMARY AND CONCLUSIONS}

In this work we put forward different ways for a direct computation of real time correlation functions in the framework of the FRG. Such approaches avoid the large systematic errors of carrying out a numerical analytic continuation of given Euclidean data by a direct computation of two-point correlation functions for Minkowski external momenta.

In the first part of the work we put forward a general FRG-framework that allows to compute Euclidean correlation functions at complex momenta for general regulators. Then, retarded correlation function can be obtained from these correlation functions in a fully numerical procedure. In particular, this involved the construction of general space-time symmetry preserving regulator functions, which avoid the occurrence of artificial regulator poles in the momentum region of interest. Such regulator functions have a broad range of possible applications not only for real time applications but also for the closely related problem of the inclusion of a finite chemical potential with $4 \mathrm{~d}$ regulator functions. They also allow to use the plethora of existing Euclidean results for real time physics. The procedure was put into practice at the example of the computation of spectral functions in the $O(N)$ model.

The second part of the work was devoted to a complementary approach starting directly within the real time formalism on the Keldysh contour. Here we focused on the formalism for the calculation of spectral functions in the closed time path formalism. Note that for a spatial flat regulator function the resulting flow equations for-

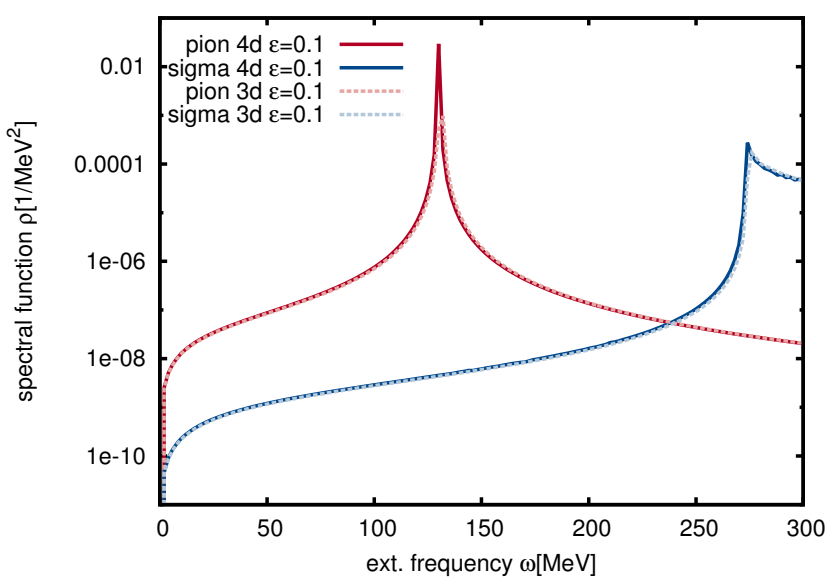

FIG. 11. Spectral functions of the $O(N)$ model at vanishing temperature comparing the result using a spatial $3 \mathrm{~d}$ regulator and a $4 \mathrm{~d}$ exponential regulator in both cases for a finite external momentum $\epsilon=0.1 \mathrm{MeV}$.

mally agree with those derived in the imaginary time formalism. We confirmed this formal equivalence, the real time representation of the flow, however, was shown to be particularly amiable towards numerical applications. The discussion focused on the formalism and applications were restricted to the $O(N)$ model as a simple, illustrative example. The extension towards general theories, and in particular the inclusion of quarks is straightforward. Furthermore, the formalism put forward in this work is directly applicable to nontrivial frequency dependencies.

\section{ACKNOWLEDGMENTS}

Acknowledgments We thank R. Alkofer, N. Christiansen, W.-J. Fu and L. von Smekal for discussions. This work is supported by the Helmholtz Alliance HA216/EMMI and the grant ERC-AdG-290623.

\section{Appendix A: General-purpose regulators for complex momenta}

We consider regulators of the form

$$
R_{k ; \Delta m_{r}^{2}}\left(p^{2}\right)=\left.\Gamma_{k}^{(2)}\left(p^{2}\right)\right|_{\phi=\phi_{0}} r\left(\frac{p^{2}+\Delta m_{r}^{2}}{k^{2}}\right),
$$

with shape function $r$, which are proportional to $\Gamma_{k}^{(2)}$ at some specific field value $\phi_{0}$. Hence at least at this particular field value $\phi_{0}$ regularised inverse propagator $\Gamma_{k}^{(2)}+\left.R_{k}\right|_{\phi_{0}}$ shares the zeros of $\Gamma_{k}^{(2)}$. Such regulators are obviously well suited to be used in connection with Taylor expansions (at $\left.\phi_{0}\right)$. In this paper we will consider exponential regulator functions as given in $(23)$. 
The above regulator functions are perfectly suited for applications where the squared mass parameter stays positive, e.g. for a Taylor expansion at the scaledependent minimum. However, both in grid implementations as well as for Taylor expansions at a fixed expansion point 32 the requirement of a positive squared mass parameter is not always satisfied. This is obviously problematic for regulators of the form (A1) as it leads to a pole in $G$. Therefore we also consider slight generalisations of Eq. (A1) i.e. modified regulators of the form

$$
R_{k ; \Delta m_{r}^{2}}\left(p^{2}\right)=\left(\left.\Delta \Gamma_{k}^{(2)}\left(p^{2}\right)\right|_{\phi=\phi_{0}}+\Delta m_{r}^{2}\right) r\left(\frac{p^{2}+\Delta m_{r}^{2}}{k^{2}}\right),
$$

that depend only on the momentum-dependent part, $\Delta \Gamma_{k}^{(2)}(p)=\Gamma_{k}^{(2)}(p)-\Gamma_{k}^{(2)}(0)$, to circumvent problems for $m^{2}<0$, and introduces an additional $\Delta m_{r}^{2} \cdot r$-term which avoids the dropping of the effective cutoff scale to zero for $\Delta m_{r}^{2} \gg k^{2}$, see App. A5. This choice of regulator obviously implies that even at $\phi_{0}$ the physical poles of $G$ and those of the regularised propagator $\left(\Gamma_{k}^{(2)}+R_{k ; \Delta m_{r}^{2}(k)}\right)^{-1}$ no longer coincide. This is however not an important requirement as long as we assure that no regulator poles occur inside $\mathcal{S}_{p_{0}, \max }$.

Independent of the chosen implementation, the overall picture is the same in both cases. One introduces an additional mass term in the regulator shape function in order to shift the additional regulator poles outside the momentum region of interest, here given by $\mathcal{S}_{p_{0}, \max }$. For large RG-scales these will be outside the strip but it requires a finite $\Delta m_{r}^{2}$ at least towards the IR to ensure that this remains the case during the full RG-flow. The detailed discussion of the constraints on the $\Delta m_{r}^{2}$-term can be found in App. A3.

\section{Constant $\Delta m_{r}^{2}$}

The simplest way of implementing the pole constraints discussed in the previous section is to use a sufficiently large constant $\Delta m_{r}^{2}$. As the $\Delta m_{r}^{2}$-term is already present at the UV cutoff scale $\Lambda_{\mathrm{UV}}$ one has to take into account the change in the initial conditions in the UV in order to directly compare to results with $\Delta m_{r}^{2}=0$. In the following we describe two procedures for obtaining twopoint functions at real time momenta starting from given initial conditions at $\Delta m_{r}^{2}=0$. The first possibility is to use a two-step procedure: Given initial conditions at $\Lambda$ for $\Delta m_{r}^{2}=0$, the proper initial condition for $\Delta m_{r}^{2} \neq 0$ is obtained by integrating a flow equation in $\Delta m_{r}^{2}$,

$$
\begin{aligned}
& \Gamma_{\Lambda ; \Delta m_{r}^{2}}^{(2)}(q+\mathrm{i} p)=\Gamma_{\Lambda ; 0}^{(2)}(q+\mathrm{i} p) \\
& +\frac{1}{2} \frac{\delta^{2}}{(\delta \phi)^{2}}\left(\int_{0}^{\Delta m_{r}^{2}} \mathrm{~d} \Delta m_{r}^{2 \prime}\left(\partial_{\Delta m_{r}^{2 \prime}} R_{\Lambda ; \Delta m_{r}^{2}}\right) G_{\Lambda ; \Delta m_{r}^{2 \prime}}\right)(q+\mathrm{i} p),
\end{aligned}
$$

where the first term is obtained by a trivial continuation of $\Gamma_{\Lambda ; 0}^{(2)}$. This procedure has to be carried out for the effective potential and the two-point function for every value of $p_{0}$. Note that for the two-point function the diagrams in (A3) have to be evaluated with the same frequency routing as the flow itself: $G \partial_{\Delta m_{r}^{2}} R_{\Lambda ; \Delta m_{r}^{2}} G(q)$ only depends on the loop frequency. As a second step, having integrated this equation to sufficiently large $\Delta m_{r}^{2} \sim p_{0, \max }^{2}$, one can now solve the flow equation in $k$,

$$
\Gamma_{k ; \Delta m_{r}^{2}}^{(2)}(p)=\Gamma_{\Lambda ; \Delta m_{r}^{2}}^{(2)}+\int_{\Lambda}^{0} d k \partial_{k} \Gamma_{k, \Delta m_{r}^{2}}^{(2)} .
$$

As an alternative to the direct integration in A4 one can make use of an equation for $\partial_{p} \Gamma_{k, \Delta m_{r}^{2}}^{(2)}(p)$ in order to successively enter the complex plane starting from the real axis. Therefore we assume a momentum-independent $\Delta m_{r}^{2}, \partial_{q} \Delta m_{r}^{2}=0$. Then we can write the flow equation for $\partial_{p} \Gamma_{k ; \Delta m_{r}^{2}}^{(2)}(p)$ as

$$
\begin{aligned}
\partial_{t} \partial_{p} \Gamma_{k}^{(2)}(p) & =\operatorname{Tr}_{q} G \partial_{t} R G(q) \partial_{p} G(q+\mathrm{i} p) \\
& =-\operatorname{Tr}_{q} G \partial_{t} R G(q)\left[G\left(\partial_{p} G^{-1}\right) G\right](q+\mathrm{i} p),
\end{aligned}
$$

where we suppressed the dependence on $\Delta m_{r}^{2}$ in order to simplify the notation. At a given step $p$, this evolution equation involves only known objects, namely $\left(G \partial_{t} R G\right)(q), \partial_{p} G^{-1}(q+\mathrm{i} p)=\left(\partial_{p} \Gamma_{k}^{(2)}+\partial_{p} R\right)(q+\mathrm{i} p)$ and $G(q+\mathrm{i} p)$. This is a very clear demonstration of how one can gradually enter the complex momentum plane starting from data on the real axis.

\section{Scale-dependent $\Delta m_{r}^{2}(k)$}

The second approach towards satisfying the pole constraints is to introduce a scale-dependent $\Delta m_{r}^{2}$-term which only sets in later during the RG flow. At the same time this circumvents the necessity of calculating modified initial conditions in order to connect to calculations at $\Delta m_{r}^{2}=0$ as the first part of two-step procedure introduced in the previous section as the $\Delta m_{r}^{2}$-term vanishes at the UV cutoff scale. An appropriate scale-dependent $\Delta m_{r}^{2}$-term can be modeled by means of a smooth theta function, i.e. using the Ansatz

$$
\Delta m_{r}^{2}(k)=\left|p_{0, \max }\right|^{2} \theta_{\alpha, \beta}\left(\left|p_{0, \max }\right| / k\right),
$$

where

$$
\theta_{\alpha, \beta}(x)=\frac{\alpha}{1+\left(\frac{\beta}{x}\right)^{n}},
$$

denotes a smooth approximation to a step function with appropriately chosen constants $\alpha, \beta$ and $n \in \mathbb{N}$, see App. A3 for further details. 


\section{Pole constraints on $\Delta m_{r}^{2}$}

In this appendix we analyse the poles of the regularised propagator $G\left(p^{2}\right)=\left(\Gamma_{k}^{(2)}\left(p^{2}\right)+R_{k}\right)^{-1}$ in more detail. In the following we consider regulators of the general form A2). A similar analysis goes through for regulators of the form A1.

We are interested in the poles of the regularised propagator or equivalently in the zeros of

$$
\left(p^{2}+\Delta m_{r}^{2}\right)(1+r)-\Delta m_{r}^{2}+m^{2}=0,
$$

which reduces for the exponential shape function 23 and $x \neq 0$ to the condition

$$
\left(e^{x^{m}}-1\right)\left(x+M^{2}\right)+x^{m}=0,
$$

for $x=\left(p^{2}+\Delta m_{r}^{2}\right) / k^{2}$ and $M^{2}=\left(m^{2}-\Delta m_{r}^{2}\right) / k^{2}$. It remains to analyse the solutions of Eq. A9 for fixed $M^{2}$. Leaving aside the case $m=1$, for $M^{2}>-1$ the equation admits a negative solution and for $M^{2}<-1$ two purely imaginary complex conjugate physical solutions $x_{\text {phys }}$. In addition there is an infinite sequence of poles introduced by the regulator. As shown exemplarily for the double exponential regulator $m=2$ in Fig. 3 for fixed finite $M^{2}$ these can be determined numerically. For asymptotically large values $M^{2} \rightarrow \pm \infty$ they converge towards the poles of the function $x(1+r(x))$ i.e. where $x^{m}=2 \pi \mathrm{i} N$ for $N \in$ $\mathbb{N}$. This gives us access the solutions $x_{\text {reg }}$ of $A 9$. On the other hand the restriction of the external momenta to the domain $\mathcal{S}_{p_{0, \max }}$, i.e. $p=\left(p_{0, R}+\mathrm{i} p_{0, I}, \vec{p}\right)$ for $\left|p_{0, I}\right|<p_{0, \max }$ and $p_{0, R} \in \mathbb{R}$, corresponds to a parabola in the complex $x$-plane bounded by

$$
\left\{t^{2}-p_{0, \max }^{2}+\vec{p}^{2}+\Delta m_{r}^{2}+2 \mathrm{i} t p_{0, \max } \mid t \in \mathbb{R}\right\} .
$$

Here we already included a $\Delta m_{r}^{2}$-term which adds to the real part and shifts the parabola in the direction of the positive real axis. Given a regulator pole at $x_{\mathrm{reg}}=x_{R}+$ $\mathrm{i} x_{I}$ it is then simple to evaluate the condition

$$
\Delta m_{r}^{2}(k) \geq p_{0, \max }^{2}-\vec{p}^{2}+k^{2} x_{R}-\frac{k^{4}}{4} \frac{x_{I}^{2}}{p_{0, \max }^{2}},
$$

which is obtained by equating real and imaginary parts of A10 to $x_{R}$ or $x_{I}$ respectively. This condition keeps the given pole outside the integration domain by shifting the parabola to the right. Putting together the constraints from the different poles it remains to parameterise an appropriate function which satisfies all of them, using the simple Ansatz $\mathrm{A} 6$ for a scale-dependent $\Delta m_{r}^{2}(k)$ or alternatively using a constant $\Delta m_{r}^{2}$ as discussed in App. A 1 . This procedure is illustrated exemplarily for the double exponential regulator, $m=2$, and $p_{0, \max }=0.33 \Lambda_{\mathrm{UV}}$ in Fig. 4. The parameter $M^{2}$ serves as an external parameter in this analysis and one has to select the value for $M^{2}$ which constrains $\Delta m_{r}^{2}$ most strongly.
Summarising the discussion of this appendix, it is always possible to prevent regulator poles from entering the domain $\mathcal{S}_{p_{0, \max }}$ at a given scale $k$ by introducing a sufficiently large term $\Delta m_{r}^{2}(k)$.

\section{Application to the $O(N)$ model: Flow equations and numerical procedure}

In this appendix we discuss the flow equations and the numerical solution procedure that lead to the numerical results on the mesonic spectral functions presented in Sec. IIC. In the truncation presented here the momentum- and complex-frequency-dependent twopoint functions are solved for on the basis of a given solution for the effective potential that serves as input for the three-point vertices occurring in the flow equation for the two-point function. In the LPA the flow equation for the effective potential takes the form

$$
\partial_{t} V_{k}\left(\phi^{2}\right)=\frac{1}{2} I^{(1)}\left(m_{\sigma}^{2}\right)+\frac{N-1}{2} I^{(1)}\left(m_{\pi}^{2}\right),
$$

where $m_{\pi}^{2}=2 V_{k}^{\prime}, m_{\sigma}^{2}=2 V_{k}^{\prime}+4 \phi^{2} V_{k}^{\prime \prime}$ and

$$
I^{(n)}\left(m^{2}\right)=\bigvee_{q} \frac{\partial_{t} R_{k ; \Delta m_{r}^{2}}\left(q^{2}\right)}{\left(q^{2}+m^{2}+R_{k ; \Delta m_{r}^{2}}\left(q^{2}\right)\right)^{n}},
$$

which was evaluated numerically for the regulator 22. The flow equation for the Euclidean two-point functions, see Fig. 12, is given by 8 ]

$$
\begin{aligned}
\partial_{t} \Gamma_{\pi}^{(2)}(p)= & \left(\Gamma_{\sigma \pi \pi}^{(3)}\right)^{2}\left(J_{\sigma \pi}(p)+J_{\pi \sigma}(p)\right) \\
& + \text { tadpole-terms } \\
\partial_{t} \Gamma_{\sigma}^{(2)}(p)= & (N-1)\left(\Gamma_{\sigma \pi \pi}^{(3)}\right)^{2} J_{\pi \pi}(p)+\left(\Gamma_{\sigma \sigma \sigma}^{(3)}\right)^{2} J_{\sigma \sigma}(p) \\
& + \text { tadpole-terms },
\end{aligned}
$$

with $\Gamma_{\sigma \pi \pi}^{(3)}$ and $\Gamma_{\sigma \sigma \sigma}^{(3)}$ given in $\mathrm{B} 35$ and

$$
\begin{aligned}
J_{i j}(p)= & \bigvee_{q} \partial_{t} R_{k ; \Delta m_{r}^{2}}\left(q^{2}\right)\left(q^{2}+m_{j}^{2}+R_{k ; \Delta m_{r}^{2}}\left(q^{2}\right)\right)^{-2} \\
& \times\left((q+p)^{2}+m_{i}^{2}+R_{k ; \Delta m_{r}^{2}}\left((q+p)^{2}\right)\right)^{-1} .
\end{aligned}
$$

The frequency routing is chosen such that the cutoff line only depends on the loop frequency $q_{0}$, that is $G \dot{R} G(q)$. As in 31] we only resolve the genuine momentum dependence of the propagator $\Delta \Gamma^{(2)}$, i.e. we solve flow equations $\partial_{t} \Delta \Gamma^{(2)}(p) \equiv \partial_{t} \Gamma^{(2)}(p)-\partial_{t} \Gamma^{(2)}(0)$, for which the tadpole terms, which are momentum-independent in our truncation, cancel. The full propagator is then obtained via

$$
\Gamma_{i}^{(2)}(p)=\Delta \Gamma_{i}^{(2)}(p)+\frac{\partial^{2}}{\left(\partial \phi_{i}\right)^{2}} V\left(\phi^{2}\right)
$$




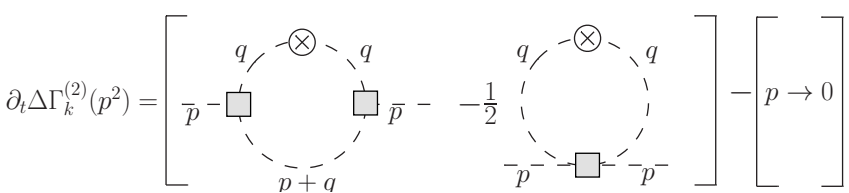

FIG. 12. Flow equation for the momentum-dependent part of the inverse propagator.

We use 22 in A14 to set up flow equations for the real and imaginary parts of the retarded propagator, keeping a fixed finite parameter $\epsilon$. Note that in addition to this contribution it remains to track the positions of the physical poles and to add the corresponding contributions involving residues at their positions, see the extensive discussion in Sec. IA

\section{Effective cutoff scales}

The inclusion of $\Delta m_{r}^{2}(k)$ in the regulator's shape function, as discussed in App. A2, leads to a modification of the effective cutoff scale with important physical consequences. One way of defining such an effective cutoff scale is to consider the gap in the propagator, i.e.

$$
k_{\text {eff;min }}^{2}(k)=\min _{p}\left(\Gamma_{k}^{(2)}\left(p^{2}\right)+R_{k}\left(p^{2}\right)\right) .
$$

Note that (A17) defines a physical cutoff scale which does not go to zero in a massive theory. In the massless limit for regularised propagators that depend monotonously on momentum (A17) coincides with the effective cutoff scale $k_{\text {eff; } R(0)}$ defined via

$$
k_{\mathrm{eff} ; R(0)}^{2}(k)=\lim _{p^{2} \rightarrow 0} R_{k ; \Delta m_{r}^{2}}\left(p^{2}\right)=\Delta m_{r}^{2} r\left(\Delta m_{r}^{2} / k^{2}\right),
$$

which can be easily evaluated for a given $\Delta m_{r}^{2}(k)$. $k_{\text {eff; } R(0)}^{2}$ tends to $k^{2}$ for $\Delta \hat{m}_{r}^{2} \equiv \Delta m_{r}^{2} / k^{2} \ll 1$ and it drops exponentially with $\Delta \hat{m}_{r}^{2}$ in the regime where $\Delta \hat{m}_{r}^{2} \gg 1$. An alternative definition is given by $k_{\text {eff; } R=\Delta \Gamma^{(2)}}$ which defines the effective cutoff scale as the momentum scale where the regulator gets of the order of the propagator itself, i.e.

$$
R_{k ; \Delta m_{r}^{2}(k)}\left(p^{2}\right)=\left.\Delta \Gamma_{k}^{(2)}\left(p^{2}\right)\right|_{p^{2}=k_{\mathrm{eff} ; R=\Delta \Gamma^{(2)}}^{2}},
$$

where $\Delta \Gamma_{k}^{(2)}\left(p^{2}\right)=\Gamma_{k}^{(2)}\left(p^{2}\right)-\Gamma_{k}^{(2)}(0)$. For $\Delta m_{r}^{2}=0$ and the double exponential regulator, $m=2, k_{\text {eff; } R=\Delta \Gamma^{(2)}} \approx$ $0.86 k$. As visible from Fig. 5 both definitions show the exponential drop of the effective cutoff scale in the regime where $\Delta \hat{m}_{r}^{2} \gg 1$.

The relation $k\left(k_{\text {eff }}\right)$ can now be inverted at least numerically in order to yield a relation $k\left(k_{\text {eff }}\right)$, which in turn could be used to rewrite the flow equation in terms of $k_{\text {eff }}$ for numerical convenience. Effective cutoff scales

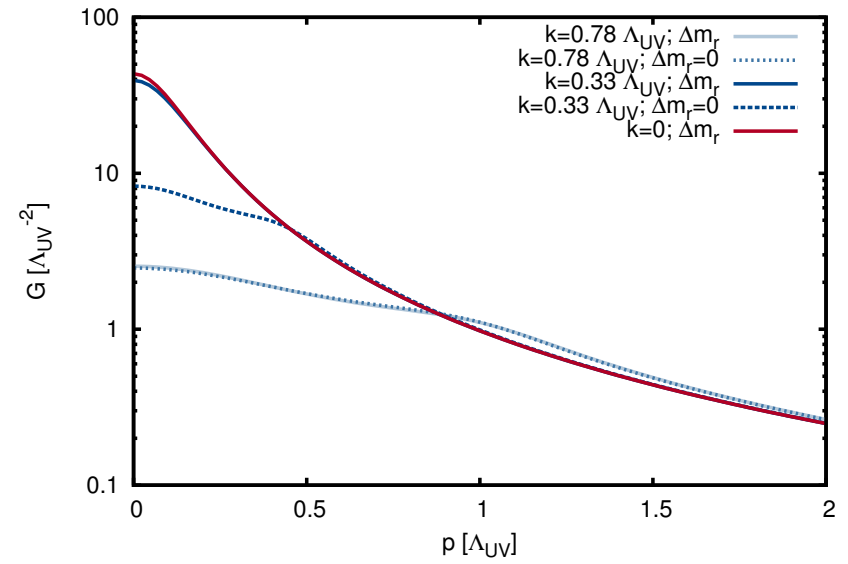

FIG. 13. Regularised pion propagator at different cutoff scales in a calculation with (solid) and without (dashed) $\Delta m_{r}^{2}$-term using the same parameter set as in Fig. 4 in comparison to the result at $k=0$.

as defined in this appendix can be used to adjust relative cutoff scales for fermions and bosons and theories with bosonic and fermionic species.

We illustrate the different effective cutoff scales at the example of the scale dependence of the regularised pion propagator shown in Fig. 13 comparing the results of a calculation with and without $\Delta m_{r}^{2}$-term. For large cutoff scales $\Delta m_{r}^{2}$ is practically zero, the effective cutoff scale coincides with the cutoff scale $k$ and both propagators agree. At smaller cutoff scales where $\Delta m_{r}^{2}$ takes a nonvanishing value, the propagators start to deviate. Note in particular that the $\Delta m_{r}^{2}$-propagator reaches its IR value already at a finite value of the cutoff scale as a result of the exponential drop of the effective cutoff scale with $\Delta \hat{m}_{r}^{2}$, see Fig. 5 .

\section{Appendix B: Flow equations in the CTP formalism}

In this appendix we discuss the derivation of the flow equations for the effective potential and the spectral functions in the CTP formalism in detail.

\section{CTP formalism}

Before discussing the CTP flow equations we start by fixing the formalism and conventions used in this work. The main object in the following is the matrix-valued 
two-point function with components defined by

$$
\begin{aligned}
& G_{+-}(x, y)=-\mathrm{i}\langle\phi(x) \phi(y)\rangle, \\
& G_{-+}(x, y)=-\mathrm{i}\langle\phi(y) \phi(x)\rangle, \\
& G_{++}(x, y)=-\mathrm{i}\langle\mathcal{T} \phi(x) \phi(y)\rangle, \\
& G_{--}(x, y)=-\mathrm{i}\langle\tilde{\mathcal{T}} \phi(y) \phi(x)\rangle,
\end{aligned}
$$

where $\mathcal{T}(\tilde{\mathcal{T}})$ denotes (anti-)time ordering. In thermal equilibrium and in momentum space the propagators are given solely in terms of the spectral function, c.f. [36] for the free case,

$$
\begin{aligned}
G_{ \pm \pm}(p)= & \pm \mathcal{P} \int_{-\infty}^{\infty} \frac{\mathrm{d} \omega}{2 \pi} \frac{\rho(\omega, \vec{p})}{p_{0}-\omega} \\
& -\mathrm{i}\left(n\left(p_{0}\right)+\frac{1}{2}\right) \rho\left(p_{0}, \vec{p}\right), \\
G_{+-}(p)= & -\mathrm{i} n\left(p_{0}\right) \rho(p), \\
G_{-+}(p)= & -\mathrm{i}\left(n\left(p_{0}\right)+1\right) \rho(p),
\end{aligned}
$$

where $\mathcal{P}$ denotes the Cauchy principal value and $n$ the bosonic thermal distribution function.

In order to calculate spectral functions we require flow equations for the real and imaginary parts of the inverse diagonal propagator $\bar{\Gamma}^{(2)}(p)$ [56]. These are related to $\Gamma_{++}^{(2)}(p)$ and $\Gamma_{+-}^{(2)}(p)$ via relations obtained in analogy to [23] using [57] to diagonalise the CTP propagator and self-energy

$$
\begin{aligned}
\operatorname{Re} \Gamma_{++}^{(2)}(p) & =\operatorname{Re} \bar{\Gamma}^{(2)}(p), \\
\operatorname{Im} \Gamma_{++}^{(2)}(p) & =\operatorname{sign}\left(p^{0}\right)\left(1+2 n\left(p^{0}\right)\right) \operatorname{Im} \bar{\Gamma}^{(2)}(p), \\
\Gamma_{+-}^{(2)}(p) & =-\mathrm{i} \operatorname{sign}\left(p^{0}\right) 2 n\left(p_{0}\right) \operatorname{Im} \bar{\Gamma}^{(2)}(p) .
\end{aligned}
$$

The real and imaginary parts of the diagonalised real time two-point function are related to the retarded correlation function via [23, 57]

$$
\begin{aligned}
& \operatorname{Re} \Gamma_{\mathrm{R}}^{(2)}(p)=\operatorname{Re} \bar{\Gamma}^{(2)}(p) \\
& \operatorname{Im} \Gamma_{\mathrm{R}}^{(2)}(p)=\operatorname{sign}\left(p_{0}\right) \operatorname{Im} \bar{\Gamma}^{(2)}(p) .
\end{aligned}
$$

Now consider the retarded propagator that, following (B2), is given by

$$
\begin{aligned}
G_{\mathrm{R}}(p) & =G_{++}(p)-G_{+-}(p) \\
& =\int_{-\infty}^{\infty} \frac{\mathrm{d} \omega}{2 \pi} \frac{\rho(\omega, \vec{p})}{p^{0}-\omega+\mathrm{i} \epsilon} \\
& =\mathcal{P} \int_{-\infty}^{\infty} \frac{\mathrm{d} \omega}{2 \pi} \frac{\rho(\omega, \vec{p})}{p^{0}-\omega}-\frac{\mathrm{i}}{2} \rho\left(p^{0}, \vec{p}\right) .
\end{aligned}
$$

This immediately implies that

$$
\begin{aligned}
\rho(p) & =-2 \operatorname{Im} G_{\mathrm{R}}(p)=-2 \operatorname{Im}\left[\Gamma_{\mathrm{R}}^{(2)}\right]^{-1} \\
& =\frac{2 \operatorname{Im} \Gamma_{\mathrm{R}}^{(2)}(p)}{\left(\operatorname{Im} \Gamma_{\mathrm{R}}^{(2)}(p)\right)^{2}+\left(\operatorname{Re} \Gamma_{\mathrm{R}}^{(2)}(p)\right)^{2}},
\end{aligned}
$$

and leaves us with the determination of flow equations for $\operatorname{Re} \Gamma_{++}^{(2)}$ and for example $\Gamma_{+-}^{(2)}$ in order to obtain the spectral function.

\section{CTP flow equation}

In this appendix we fix the conventions that are required to derive the CTP flow equation. These are chosen such that the functional relations between the different generating functionals stay as close to the Euclidean relations as possible. We start by defining a generating functional

$$
\begin{aligned}
Z_{k}\left[J_{+}, J_{-}\right]= & \int \mathcal{D} \varphi_{+} \mathcal{D} \varphi_{-} \exp \left[\mathrm{i} S\left[\varphi_{+}, \varphi_{-}\right]\right. \\
& \left.+\mathrm{i} \Delta S_{k}\left[\varphi_{+}, \varphi_{-}\right]-\mathrm{i} \int_{x} \varphi_{a}(x) \eta^{a b} J_{b}(x)\right], \\
\Delta S_{k}\left[\varphi_{+}, \varphi_{-}\right]= & \frac{1}{2} \int_{x, y} \varphi_{a}(x) \eta^{a b}\left(R_{k}\right)_{b c}(x, y) \eta^{c d} \varphi_{d}(y),
\end{aligned}
$$

where $\eta=\operatorname{diag}(1,-1)$ and $a, b, c, d \in\{+,-\}$. Defining the generating functional for connected Greens functions $W_{k}$ and the effective action $\Gamma_{k}$ via

$$
\begin{aligned}
& W_{k}\left[J_{+}, J_{-}\right]=\mathrm{i} \log Z_{k}\left[J_{+}, J_{-}\right], \\
& \Gamma_{k}\left[\phi_{+}, \phi_{-}\right]=J_{a} \eta^{a b} \phi_{b}-W_{k}-\Delta S_{k}\left[\phi_{+}, \phi_{-}\right],
\end{aligned}
$$

leads to the relations

$$
\begin{array}{r}
\frac{\delta W_{k}}{\delta J_{a}}=\eta^{a b} \phi_{b}, \\
\frac{\delta\left(\Gamma_{k}+\Delta S_{k}\right)}{\delta \phi_{a}}=\eta^{a b} J_{b},
\end{array}
$$

and finally to the flow equation

$$
\begin{aligned}
\partial_{t} \Gamma_{k} & =\frac{\mathrm{i}}{2} \operatorname{Tr}\left(\frac{1}{\Gamma_{k}^{(2)}+R_{k}}\right)_{a b}\left(\partial_{t} R_{k}\right)_{b a} \\
& =\frac{\mathrm{i}}{2} \operatorname{Tr} G_{a b}\left(\partial_{t} R_{k}\right)_{b a} .
\end{aligned}
$$

\section{Flow equation for the effective potential}

As a first step we consider the flow equation for the effective potential in the CTP formalism. Note that evalu- 
ating $\Gamma$ for constant fields $\phi^{+}=\phi^{-}=\phi$ yields a contribution with a vanishing real part. In order to project onto the effective potential we take the real part after taking a single $\phi^{+}$-derivative and setting fields $\phi^{+}=\phi^{-}=\phi$ to constant afterwards. We find

$$
\partial_{\phi^{+}} \partial_{t} \Gamma_{k}=\tilde{\partial}_{t} \frac{\mathrm{i}}{2} \operatorname{Tr} G_{a b} \Gamma_{b a+},
$$

with the partial $t$-derivative at fixed $\Gamma_{k}^{(n)}$,

$$
\tilde{\partial}_{t}=\left.\partial_{t}\right|_{\Gamma_{k}^{(n)}}=\operatorname{Tr} \dot{R}_{k} \frac{\delta}{\delta R_{k}}=\sum_{a= \pm} \operatorname{Tr} \dot{R}_{k}^{a} \frac{\delta}{\delta R_{k}^{a}} .
$$

In the following we resort to the canonical choice $R_{k}^{+}=$ $-R_{k}^{-}=R_{k}$ and restrict ourselves to the effective potential. Now we have

$$
\partial_{t} \partial_{\phi} V_{k}\left(\phi^{2}\right)=-\frac{1}{2} \dot{R}_{k} \partial_{R_{k}} \operatorname{Tr}\left(\operatorname{Im} G_{a b} \Gamma_{b a+}^{(3)}\right),
$$

with $a, b= \pm$. The local potential approximation (LPA) as simplest approximation involves inserting free spectral functions

$$
\begin{aligned}
\rho\left(p_{0}, \vec{p}\right) & =(2 \pi) \operatorname{sign}\left(p_{0}\right) \delta\left(-p_{0}^{2}+\vec{p}^{2}+m^{2}\right) \\
& =\frac{2 \pi}{2 \epsilon_{p}}\left(\delta\left(p_{0}-\epsilon_{p}\right)-\delta\left(p_{0}+\epsilon_{p}\right)\right),
\end{aligned}
$$

with $\epsilon_{p}$ from (7). In the LPA approximation we have a deformed classical dispersion with a field-dependent mass as given by

$$
m_{k}^{2}\left(\phi^{2}\right)=2 V_{k}^{\prime}\left(\phi^{2}\right)+4 \phi^{2} V_{k}^{\prime \prime}\left(\phi^{2}\right),
$$

for a single field mode, where primes denote derivatives with respect to $\phi^{2}$. Correspondingly for the regulator (26), the spectral function (B14) generalises to

$$
\begin{aligned}
& \rho(\omega, \vec{p})=(2 \pi) \operatorname{sign}\left(p_{0}\right) \\
& \times \delta\left(-p_{0}^{2}+\vec{p}^{2}+m_{k}^{2}\left(\phi^{2}\right)\right. \\
&\left.+R_{k}\left(-p_{0}^{2}+\vec{p}^{2}\right)\left(r_{0}\left(p_{0}^{2} / k^{2}\right)+r_{s}\left(\vec{p}^{2} / k^{2}\right)\right)\right) .
\end{aligned}
$$

Here, we restrict ourselves to spatial regulators. In the simplest case of a spatial regulator function, i.e. for $R_{k}\left(p^{2}\right) \equiv k^{2}, r_{0} \equiv 0$ and $r_{s}(x)=x r(x)$ in $(26)$, we can trivially perform the frequency integration and the flow equation for the effective potential now takes the simple form

$$
\begin{aligned}
\partial_{t} \partial_{\phi} V_{k}(\phi) & =\frac{1}{2} \int_{\vec{q}} \dot{r} \partial_{r} \frac{1+2 n\left(\epsilon_{q}\right)}{2 \epsilon_{q}} \Gamma_{\phi \phi \phi}^{(3)} \\
& =\frac{1}{2} \int_{\vec{q}} \vec{q}^{2} \dot{r} \partial_{\epsilon_{q}}\left(\frac{1+2 n\left(\epsilon_{q}\right)}{2 \epsilon_{q}}\right) \frac{\partial \epsilon_{q}}{\partial \phi} \\
& =\partial_{\phi} \frac{1}{2} \int_{\vec{q}} \frac{\vec{q}^{2} \dot{r}}{2 \epsilon_{q}}\left(1+2 n\left(\epsilon_{q}\right)\right),
\end{aligned}
$$

with

$$
\epsilon_{q}\left(\phi^{2}\right)=\sqrt{\vec{q}^{2}\left(1+r\left(\vec{q}^{2} / k^{2}\right)\right)+m_{k}^{2}\left(\phi^{2}\right)} .
$$

The flow equation (B17) is nothing but the derivative of the standard flow equation for the effective action/potential for a three-dimensional spatial regulator. In particular, for the flat regulator, the $3 \mathrm{~d}$-analogue of the LPA-optimised regulator [58, where $r(x)=(-1+$ $1 / x) \theta(1-x)$, we arrive at

$$
\partial_{t} \partial_{\phi} V_{k}\left(\phi^{2}\right)=\partial_{\phi}\left(\frac{k^{5}}{6 \pi^{2}} \frac{\operatorname{coth}\left(\frac{\sqrt{k^{2}+m_{k}^{2}\left(\phi^{2}\right)}}{2 T}\right)}{\sqrt{k^{2}+m_{k}^{2}\left(\phi^{2}\right)}}\right),
$$

the well-known flow for the spatial flat regulator as derived in the imaginary time formalism.

\section{Flow equations for spectral functions}

In the CTP formalism with spatial regulator functions the direct computation of real time momentum and frequency dependence is straightforward. Note however, that the regulator explicitly breaks Lorentz invariance and frequency integrations only have polynomially decaying integrands.

For $R_{k}^{+}=-R_{k}^{-}=R_{k}$ again, we find the symbolic flow equations for the different components of the two-point function

$$
\begin{aligned}
\partial_{t} \Gamma_{++}^{(2)}(p)= & -\frac{\mathrm{i}}{2} \tilde{\partial}_{t} \operatorname{Tr}\left(\Gamma_{+}^{(3)} G_{++} \Gamma_{+}^{(3)} G_{++}\right) \\
& +\frac{\mathrm{i}}{2} \tilde{\partial}_{t} \operatorname{Tr}\left(\Gamma_{+}^{(4)} G_{++}\right), \\
\partial_{t} \Gamma_{+-}^{(2)}(p)= & -\frac{\mathrm{i}}{2} \tilde{\partial}_{t} \operatorname{Tr}\left(\Gamma_{+}^{(3)} G_{+-} \Gamma_{-}^{(3)} G_{-+}\right) .
\end{aligned}
$$

Evaluating (B21) and neglecting the tadpole terms leads to

$$
\begin{aligned}
\partial_{t} & \operatorname{Re} \Gamma_{++}^{(2)}(p) \\
=+ & \frac{1}{2} \tilde{\partial}_{t} \int_{q} \Gamma_{+}^{(3)} \operatorname{Im} G_{++}(q+p) \Gamma_{+}^{(3)} \operatorname{Re} G_{++}(q) \\
+ & \frac{1}{2} \tilde{\partial}_{t} \int_{q} \Gamma_{+}^{(3)} \operatorname{Re} G_{++}(q+p) \Gamma_{+}^{(3)} \operatorname{Im} G_{++}(q) \\
=- & \frac{1}{2} \tilde{\partial}_{t} \int_{\vec{q}} \frac{\Gamma_{+}^{(3)} \Gamma_{+}^{(3)}}{4 \epsilon_{q} \epsilon_{q+p}}\left[\left(n\left(\epsilon_{q+p}\right)-n\left(\epsilon_{q}\right)\right)\right. \\
& \times \mathcal{P}\left(\frac{1}{p_{0}-\epsilon_{q}+\epsilon_{q+p}}-\frac{1}{p_{0}+\epsilon_{q}-\epsilon_{q+p}}\right) \\
+ & \left(1+n\left(\epsilon_{q+p}\right)+n\left(\epsilon_{q}\right)\right) \\
& \left.\times \mathcal{P}\left(\frac{1}{p_{0}-\epsilon_{q}-\epsilon_{q+p}}-\frac{1}{p_{0}+\epsilon_{q}+\epsilon_{q+p}}\right)\right] .
\end{aligned}
$$


Evaluating B21) using the identity $\left(1+n\left(q^{0}\right)\right) n\left(q^{0}+\right.$ $\left.p^{0}\right)=n\left(p^{0}\right)\left(n\left(q^{0}\right)-n\left(q^{0}+p^{0}\right)\right)$ we find

$$
\begin{aligned}
& \partial_{t} \Gamma_{+-}^{(2)}(p) \\
= & -\frac{\mathrm{i}}{2} \tilde{\partial}_{t} \int_{q} \Gamma_{+}^{(3)} G_{+-}(q+p) \Gamma_{-}^{(3)} G_{-+}(q) \\
= & \frac{\mathrm{i}}{2} n\left(p^{0}\right) \tilde{\partial}_{t} \int_{q} \Gamma_{+}^{(3)} \Gamma_{-}^{(3)}\left(n\left(q^{0}\right)-n\left(p^{0}+q^{0}\right)\right) \rho(q+p) \rho(q)
\end{aligned}
$$

leading to

$$
\begin{aligned}
\partial_{t} \Gamma_{+-}^{(2)}(p)= & \frac{\mathrm{i}}{2} n\left(p^{0}\right) \tilde{\partial}_{t} \int_{\vec{q}} \frac{2 \pi}{4 \epsilon_{q+p} \epsilon_{q}} \Gamma_{+}^{(3)} \Gamma_{-}^{(3)} \\
& \times\left[\left(n\left(\epsilon_{q}\right)-n\left(\epsilon_{q+p}\right)\right)\right. \\
& \times\left(\delta\left(p_{0}+\epsilon_{q}-\epsilon_{q+p}\right)-\delta\left(p_{0}-\epsilon_{q}+\epsilon_{q+p}\right)\right) \\
& +\left(1+n\left(\epsilon_{q}\right)+n\left(\epsilon_{q+p}\right)\right) \\
& \left.\times\left(\delta\left(p_{0}-\epsilon_{q}-\epsilon_{q+p}\right)-\delta\left(p_{0}+\epsilon_{q}+\epsilon_{q+p}\right)\right)\right] .
\end{aligned}
$$

If one now exploits the fact that the regulator enters the flow only via $\epsilon_{s}$ one may rewrite $\tilde{\partial}_{t}$ as

$$
\int_{\vec{s}} \dot{R}(\vec{s}) \frac{\delta}{\delta R(\vec{s})}=\int_{\vec{s}} \frac{\dot{R}(\vec{s})}{2 \epsilon_{s}} \frac{\delta}{\delta \epsilon_{s}} .
$$

For general regulators one now can perform the $\epsilon$ derivative and then resolve the $\delta, \delta^{\prime}$-functions. Here we restrict ourselves again to the three-dimensional flat regulator

$$
R_{k}^{\text {flat }}\left(\vec{p}^{2}\right)=\left(k^{2}-\vec{p}^{2}\right) \theta\left(k^{2}-\vec{p}^{2}\right) .
$$

This allows us to analytically resolve the $\delta$-functions before taking the $\tilde{t}$-derivative with $\tilde{\partial}_{t} \Gamma_{k}^{(n)}=0$ for all $n \in \mathbb{N}^{+}$, i.e. which acts only on the explicit $k$ dependence of the propagators. The $\delta$-functions in (B24) read

$$
\begin{aligned}
& \delta\left(p_{0} \pm \epsilon_{q} \pm \epsilon_{q+p}\right) \\
= & \delta\left(p_{0} \pm \epsilon_{k} \pm \epsilon_{k}\right) \theta\left(k^{2}-\vec{q}^{2}\right) \theta\left(k^{2}-(\vec{q}+\vec{p})^{2}\right) \\
& +\delta\left(p_{0} \pm \epsilon_{q} \pm \epsilon_{k}\right) \theta\left(\vec{q}^{2}-k^{2}\right) \theta\left(k^{2}-(\vec{q}+\vec{p})^{2}\right) \\
& +\delta\left(p_{0} \pm \epsilon_{k} \pm \epsilon_{q+p}\right) \theta\left(k^{2}-\vec{q}^{2}\right) \theta\left((\vec{q}+\vec{p})^{2}-k^{2}\right) \\
& +\delta\left(p_{0} \pm \epsilon_{q} \pm \epsilon_{q+p}\right) \theta\left(\vec{q}^{2}-k^{2}\right) \theta\left((\vec{q}+\vec{p})^{2}-k^{2}\right),
\end{aligned}
$$

where both signs can be varied independently. The $\delta$ function in the first term on the right hand side does not depend on the loop momentum $\vec{q}$, however, the integration can readily be performed. The other three $\delta$-functions in (B27) depend on the loop momentum $q$.
They can be rewritten in terms of $\delta$-functions w.r.t. $q$ with the help of

$$
\begin{aligned}
\delta\left(p_{0}+\epsilon_{q} \pm \epsilon_{k}\right) & =\frac{\epsilon_{q} \delta\left(q-q^{(1) \pm}\right)}{q}, \\
\delta\left(p_{0}+\epsilon_{q+p} \pm \epsilon_{k}\right) & =\sum_{i=1}^{2} \frac{\epsilon_{q+p} \delta\left(q-q_{i}^{(2) \pm}\right)}{|q+p x|}, \\
\delta\left(p_{0}+\epsilon_{q} \pm \epsilon_{q+p}\right) & =\sum_{i=1}^{2} \frac{\delta\left(q-q_{i}^{(3)}\right)}{\left|\frac{q}{\epsilon_{q}} \pm \frac{(q+p x)}{\epsilon_{q+p}}\right|},
\end{aligned}
$$

where $x=\vec{q} \cdot \vec{p} /(|\vec{q}||\vec{p}|)$ and we can assume without loss of generality that the second summand appears with positive coefficient. Note that $q^{(i)}$ depend on $p_{0},|\vec{p}|, x, m_{k}^{2}, k$. The $\tilde{t}$-derivative only hits the explicit $k$ dependence and not $m_{k}^{2}$. The $q^{(i)}$ are given analytically, to wit

$$
\begin{aligned}
q^{(1) \pm}= & \sqrt{\left(p_{0} \pm \epsilon_{k}\right)^{2}-m^{2}}, \\
q_{i}^{(2) \pm}= & -|\vec{p}| x+(-1)^{i} \sqrt{\vec{p}^{2}\left(x^{2}-1\right)+\left(p_{0} \pm \epsilon_{k}\right)^{2}-m^{2}}, \\
q_{i}^{(3)}= & \frac{1}{2\left(-p_{0}^{2}+\vec{p}^{2} x^{2}\right)}\left\{-|\vec{p}|^{3} x+|\vec{p}| p_{0}^{2} x\right. \\
& \left.+(-1)^{i} p_{0} \sqrt{\left(\vec{p}^{2}-p_{0}^{2}\right)^{2}+4 m^{2}\left(-p_{0}^{2}+\vec{p}^{2} x^{2}\right)}\right\},
\end{aligned}
$$

where the set of the two roots of $q^{(3)}$ agree.

\section{Application to the $O(N)$ model}

As nontrivial example we discuss the computation of spectral functions in the $O(N)$ model on the basis of a given solution for the effective potential. As demonstrated above for a single scalar field the real time flow equation for the effective potential coincides with that derived in the imaginary time formalism. Turning to two-point functions, the application to the $O(N)$ model requires more general expressions with different particles in the loop diagrams. Therefore we consider

$$
\begin{aligned}
\bar{J}_{j i}^{\mathrm{Re}}(p)= & +\frac{1}{2} \int_{q} \operatorname{Im} G_{++}^{j}(q+p) \operatorname{Re} G_{++}^{i}(q) \\
& +\frac{1}{2} \int_{q} \operatorname{Re} G_{++}^{j}(q+p) \operatorname{Im} G_{++}^{i}(q),
\end{aligned}
$$


leading to

$$
\begin{aligned}
\bar{J}_{j i}^{\mathrm{Re}}(p)=- & \frac{1}{2} \int_{\vec{q}} \frac{1}{4 \epsilon_{q}^{i} \epsilon_{q+p}^{j}}\left[\left(n\left(\epsilon_{q}^{i}\right)-n\left(\epsilon_{q+p}^{j}\right)\right)\right. \\
& \times \mathcal{P}\left(\frac{1}{p_{0}+\epsilon_{q}^{i}-\epsilon_{q+p}^{j}}-\frac{1}{p_{0}-\epsilon_{q}^{i}+\epsilon_{q+p}^{j}}\right) \\
+ & \left(1+n\left(\epsilon_{q}^{i}\right)+n\left(\epsilon_{q+p}^{j}\right)\right) \\
& \left.\times \mathcal{P}\left(\frac{1}{p_{0}-\epsilon_{q}^{i}-\epsilon_{q+p}^{j}}-\frac{1}{p_{0}+\epsilon_{q}^{i}+\epsilon_{q+p}^{j}}\right)\right] .
\end{aligned}
$$

and similarly for the imaginary part, following (B3),

$$
\begin{aligned}
\bar{J}_{j i}^{\operatorname{Im}}(p)= & -\frac{1}{2} \frac{\operatorname{sign}\left(p^{0}\right)}{2 n\left(p_{0}\right)} \int_{q} G_{+-}^{j}(q+p) G_{-+}^{i}(q) \\
= & \frac{1}{4} \operatorname{sign}\left(p_{0}\right) \\
& \times \int_{q}\left(n\left(q^{0}\right)-n\left(q^{0}+p^{0}\right)\right) \rho^{j}(q+p) \rho^{i}(q),
\end{aligned}
$$

leading to

$$
\begin{aligned}
\bar{J}_{j i}^{\operatorname{Im}}(p)= & \pi \operatorname{sign}\left(p_{0}\right) \int_{\vec{q}} \frac{1}{8 \epsilon_{q+p}^{j} \epsilon_{q}^{i}} \\
& {\left[\left(n\left(\epsilon_{q}^{i}\right)-n\left(\epsilon_{q+p}^{j}\right)\right)\right.} \\
& \times\left(\delta\left(p_{0}+\epsilon_{q}^{i}-\epsilon_{q+p}^{j}\right)-\delta\left(p_{0}-\epsilon_{q}^{i}+\epsilon_{q+p}^{j}\right)\right) \\
& +\left(1+n\left(\epsilon_{q}^{i}\right)+n\left(\epsilon_{q+p}^{j}\right)\right) \\
& \left.\times\left(\delta\left(p_{0}-\epsilon_{q}^{i}-\epsilon_{q+p}^{j}\right)-\delta\left(p_{0}+\epsilon_{q}^{i}+\epsilon_{q+p}^{j}\right)\right)\right] .
\end{aligned}
$$

where the subscripts $i(j)$ refer to masses $m_{i}^{2}\left(m_{j}^{2}\right)$ in the respective propagators $G^{i}$, spectral functions $\rho^{i}$ and quasiparticle energies $\epsilon^{i}$. Using these definitions we can write the flow equations for the real and imaginary parts of the respective inverse propagators as

$$
\begin{aligned}
\partial_{t} \operatorname{Im} \bar{\Gamma}_{\pi \pi}^{(2)}(p)= & \left(\Gamma_{\sigma \pi \pi}^{(3)}\right)^{2} \tilde{\partial}_{t}\left(\bar{J}_{\sigma \pi}^{\operatorname{Im}}(p)+\bar{J}_{\pi \sigma}^{\operatorname{Im}}(p)\right) \\
\partial_{t} \operatorname{Re} \bar{\Gamma}_{\pi \pi}^{(2)}(p)= & \left(\Gamma_{\sigma \pi \pi}^{(3)}\right)^{2} \tilde{\partial}_{t}\left(\bar{J}_{\sigma \pi}^{\operatorname{Re}}(p)+\bar{J}_{\pi \sigma}^{\operatorname{Re}}(p)\right)+\text { const. } \\
\partial_{t} \operatorname{Im} \bar{\Gamma}_{\sigma \sigma}^{(2)}(p)= & (N-1)\left(\Gamma_{\sigma \pi \pi}^{(3)}\right)^{2} \tilde{\partial}_{t} \bar{J}_{\pi \pi}^{\operatorname{Im}}(p) \\
& +\left(\Gamma_{\sigma \sigma \sigma}^{(3)}\right)^{2} \tilde{\partial}_{t} \bar{J}_{\sigma \sigma}^{\operatorname{Im}}(p) \\
\partial_{t} \operatorname{Re} \bar{\Gamma}_{\sigma \sigma}^{(2)}(p)= & (N-1)\left(\Gamma_{\sigma \pi \pi}^{(3)}\right)^{2} \tilde{\partial}_{t} \bar{J}_{\pi \pi}^{\operatorname{Re}}(p) \\
& +\left(\Gamma_{\sigma \sigma \sigma}^{(3)}\right)^{2} \tilde{\partial}_{t} \bar{J}_{\sigma \sigma}^{\operatorname{Re}}(p)+\text { const. }
\end{aligned}
$$

identifying $\sigma \equiv 0$ and $\pi \in \mathbb{N}_{+}$in the subscripts. As above we only resolve the genuine momentum dependence of the real part of the propagator by considering the flow equations $\partial_{t} \Delta \Gamma^{(2)}(p)=\partial_{t} \Gamma^{(2)}(p)-\partial_{t} \Gamma^{(2)}(0)$, for which the momentum-independent tadpole terms cancel, to which the corresponding contribution from the effective potential is added in order to obtain the full propagator. Here we aim to evaluate the spectral functions on the basis of a given solution for the effective potential. Therefore we approximate three-point functions momentumindependently from the corresponding vertices extracted from the effective potential, i.e. explicitly via

$$
\begin{aligned}
& \Gamma_{\sigma \pi \pi}^{(3)} \equiv \Gamma_{+; \sigma \pi \pi}^{(3)}=-\Gamma_{-; \sigma \pi \pi}^{(3)}=4 V_{k}^{\prime \prime} \phi \\
& \Gamma_{\sigma \pi \pi}^{(3)} \equiv \Gamma_{+; \sigma \sigma \sigma}^{(3)}=-\Gamma_{-; \sigma \sigma \sigma}^{(3)}=12 V_{k}^{\prime \prime} \phi+8 V_{k}^{(3)} \phi^{3},
\end{aligned}
$$

where derivatives denote derivatives with respect to $\phi^{2}$. As simplest nontrivial application we are interested in zero temperature spectral functions at vanishing external three-momentum $\vec{p}=\overrightarrow{0}$. In this case we find

$$
\begin{aligned}
& \bar{J}_{j i}^{\mathrm{Re}}\left(p_{0}\right)=-\int_{\vec{q}} \frac{1}{4 \epsilon_{q}^{j} \epsilon_{q}^{i}} \mathcal{P}\left(\frac{\epsilon_{q}^{i}+\epsilon_{q}^{j}}{p_{0}^{2}-\left(\epsilon_{q}^{i}+\epsilon_{q}^{j}\right)^{2}}\right), \\
& \bar{J}_{j i}^{\mathrm{Im}}\left(p_{0}\right)=-\pi \operatorname{sign}\left(p_{0}\right) \sum_{ \pm} \int_{\vec{q}} \frac{\mp \delta\left(\epsilon_{q}^{i}+\epsilon_{q}^{j} \mp p_{0}\right)}{8 \epsilon_{q}^{j} \epsilon_{q}^{i}} .
\end{aligned}
$$

For $|\vec{q}|<k$ we have $\delta\left(\epsilon_{q}^{i}+\epsilon_{q}^{j}-p_{0}\right)=\delta\left(\epsilon_{k}^{i}+\epsilon_{k}^{j}-p_{0}\right)$, where $\epsilon_{k}^{i}=\sqrt{k^{2}+m_{i}^{2}}$, whereas for $|\vec{q}|>k$ we find

$$
\delta\left(\epsilon_{q}^{i}+\epsilon_{q}^{j}-p_{0}\right)=\frac{\delta\left(q-q_{f}\left(p_{0}, m_{i}, m_{j}\right)\right)}{\left|\frac{q}{\epsilon_{q}^{i}}+\frac{q}{\epsilon_{q}^{j}}\right|}
$$

where

$$
q_{f}\left(p_{0}, m_{i}, m_{j}\right)=\sqrt{\frac{\left(\left(m_{i}-m_{j}\right)^{2}-p_{0}^{2}\right)\left(\left(m_{i}+m_{j}\right)^{2}-p_{0}^{2}\right)}{4 p_{0}^{2}}} .
$$

It remains to evaluate the $\tilde{t}$-derivative, which acts only on the explicit $k$ dependence. Here only $|\vec{q}|<k$ contributes and we find, restricting ourselves without loss of 
generality to $p_{0}>0$,

$$
\begin{aligned}
& \tilde{\partial}_{t} \bar{J}_{j i}^{\mathrm{Re}}\left(p_{0}\right) \\
& =-\frac{k^{3}}{24 \pi^{2}} \tilde{\partial}_{t}\left(\frac{1}{\epsilon_{k}^{i} \epsilon_{k}^{j}} \mathcal{P}\left(\frac{\epsilon_{k}^{i}+\epsilon_{k}^{j}}{p_{0}^{2}-\left(\epsilon_{k}^{i}+\epsilon_{k}^{j}\right)^{2}}\right)\right) \\
& =-\frac{k^{5}}{24 \pi^{2}} \mathcal{P} \frac{\left(\epsilon_{k}^{i}+\epsilon_{k}^{j}\right)^{3}\left(\epsilon_{k}^{i 2}+\epsilon_{k}^{i} \epsilon_{k}^{j}+\epsilon_{k}^{j 2}\right)-\left(\epsilon_{k}^{i 3}+\epsilon_{k}^{j 3}\right) p_{0}^{2}}{\left.\epsilon_{k}^{i 3} \epsilon_{k}^{j} 3\left(\epsilon_{k}^{i}+\epsilon_{k}^{j}\right)^{2}-p_{0}^{2}\right)^{2}}, \\
& \tilde{\partial}_{t} \bar{J}_{j i}^{\operatorname{Im}}\left(p_{0}\right) \\
& =\frac{k^{3}}{48 \pi} \tilde{\partial}_{t}\left(\frac{\delta\left(\epsilon_{k}^{i}+\epsilon_{k}^{j}-p_{0}\right)}{\epsilon_{k}^{i} \epsilon_{k}^{j}}\right) \\
& =\frac{k^{3}}{48 \pi} \frac{1}{\epsilon_{k}^{i}+\epsilon_{k}^{j}}\left[\delta^{\prime}\left(k-k_{s}^{i j}\left(p_{0}\right)\right)-\frac{k}{\epsilon_{k}^{i} \epsilon_{k}^{j}} \delta\left(k-k_{s}^{i j}\left(p_{0}\right)\right)\right] .
\end{aligned}
$$

For the imaginary part we rewrote the occurring delta functions as delta functions in $k$ via

$$
\delta\left(\epsilon_{k}^{i}+\epsilon_{k}^{j}-p_{0}\right)=\frac{\delta\left(k-k_{s}^{i j}\left(p_{0}\right)\right)}{\frac{k}{\epsilon_{k}^{i}}+\frac{k}{\epsilon_{k}^{j}}},
$$

where $k_{s}^{i j}\left(p_{0}\right)$ is the solution of the equation

$$
\epsilon_{k_{s}^{i j}\left(p_{0}\right)}^{i}+\epsilon_{k_{s}^{i j}\left(p_{0}\right)}^{j}=p_{0} .
$$

In particular, for $\epsilon_{k}^{i} \geq m_{k=0}^{i}$ this entails that no imaginary part can build up below the threshold i.e. $p_{0} \geq$ $m_{\pi}+m_{\sigma}$ for the pion or $p_{0} \geq 2 m_{\pi}$ for the sigma meson spectral function respectively. Correspondingly the spectral functions have to vanish below these thresholds. The only exception occurs for momenta where $\operatorname{Re} \bar{\Gamma}^{(2)}(p)=\operatorname{Im} \bar{\Gamma}^{(2)}(p)=0$, see 3 . For $\vec{p}=0$ this occurs at the pole mass where $p_{0}^{2}=m_{\text {pole }}^{2}$, see 31] for a discussion of different mass definitions. Here the spectral function shows a delta function. As it is obvious from (B39), the imaginary part can be computed without even explicitly carrying out an integration in $k$.

\section{Equivalence of real and imaginary time flows}

These results can be compared to spectral functions obtained from Euclidean calculations with complex ex- ternal momenta using spatial regulator functions [8] in an otherwise identical truncation. In fact, the flow equations put forward here turn out to be formally equivalent to the ones put forward in [6-10 starting from an imaginary time formalism. This equivalence is nothing but the equivalence of using the real time or the imaginary time formalism for the evaluation of one-loop diagrams [23, 55]. It is however worthwhile to illustrate the formal equivalence again for this particular example, restricting ourselves for simplicity to the case of zero temperature and vanishing spatial external momentum. We consider the expression for the loop diagram for Euclidean external momentum, which reads

$$
\begin{aligned}
\bar{J}_{j i}^{\text {eucl }}\left(p_{0}\right)= & -\frac{1}{2} \int_{q} G^{j}(q+p) G^{i}(q) \\
= & -\int_{\vec{q}} \frac{1}{4 \epsilon_{q}^{i} \epsilon_{q}^{j}} \frac{\epsilon_{q}^{i}+\epsilon_{q}^{j}}{p_{0}^{2}+\left(\epsilon_{q}^{i}+\epsilon_{q}^{j}\right)^{2}} \\
= & -\int_{\vec{q}} \frac{1}{8 \epsilon_{q}^{i} \epsilon_{q}^{j}}\left(\frac{1}{\mathrm{i} p_{0}+\epsilon_{q}^{i}+\epsilon_{q}^{j}}\right. \\
& \left.+\frac{1}{-\mathrm{i} p_{0}+\epsilon_{q}^{i}+\epsilon_{q}^{j}}\right) .
\end{aligned}
$$

Performing the analytic continuation we find using (2) and the Sokhotski-Plemelj formula

$$
\begin{aligned}
\bar{J}_{j i}^{\mathrm{R}, \mathrm{IT}}\left(p_{0}\right)= & -\lim _{\epsilon \rightarrow 0} \bar{J}_{i j}^{\mathrm{eucl}}\left(-\mathrm{i}\left(p_{0}+\mathrm{i} \epsilon\right)\right) \\
= & \mathcal{P} \int_{\vec{q}} \frac{1}{4 \epsilon_{q}^{i} \epsilon_{q}^{j}} \frac{\epsilon_{q}^{i}+\epsilon_{q}^{j}}{-p_{0}^{2}+\left(\epsilon_{q}^{i}+\epsilon_{q}^{j}\right)^{2}} \\
& +\mathrm{i} \pi \sum_{ \pm} \int_{\vec{q}} \frac{ \pm \delta\left(\mp p_{0}+\epsilon_{q}^{i}+\epsilon_{q}^{j}\right)}{8 \epsilon_{q}^{i} \epsilon_{q}^{j}}
\end{aligned}
$$

By comparison to B36 one finds

$$
\begin{aligned}
& \operatorname{Re} \bar{J}_{j i}^{\mathrm{R}, \mathrm{IT}}\left(p_{0}\right)=\bar{J}_{j i}^{\mathrm{Re}}\left(p_{0}\right) \\
& \operatorname{Im} \bar{J}_{j i}^{\mathrm{R}, \mathrm{IT}}\left(p_{0}\right)=\operatorname{sign}\left(p_{0}\right) \bar{J}_{j i}^{\mathrm{Im}}\left(p_{0}\right),
\end{aligned}
$$

which is completely consistent with (B4).
[1] M. Haas, L. Fister, and J. M. Pawlowski, Phys.Rev. D90, 091501 (2014), 1308.4960.

[2] N. Christiansen, M. Haas, J. M. Pawlowski, and N. Strodthoff, Phys. Rev. Lett. 115, 112002 (2015),
1411.7986.

[3] M. Mitter, J. M. Pawlowski, and N. Strodthoff, Phys.Rev. D91, 054035 (2015), 1411.7978. 
[4] J. Braun, L. Fister, J. M. Pawlowski, and F. Rennecke, Phys. Rev. D94, 034016 (2016), 1412.1045.

[5] S. Floerchinger, JHEP 1205, 021 (2012), 1112.4374.

[6] N. Strodthoff, B.-J. Schaefer, and L. von Smekal, Phys.Rev. D85, 074007 (2012), 1112.5401.

[7] K. Kamikado, N. Strodthoff, L. von Smekal, and J. Wambach, Phys.Lett. B718, 1044 (2013), 1207.0400.

[8] K. Kamikado, N. Strodthoff, L. von Smekal, and J. Wambach, Eur.Phys.J. C74, 2806 (2014), 1302.6199.

[9] R.-A. Tripolt, N. Strodthoff, L. von Smekal, and J. Wambach, Phys.Rev. D89, 034010 (2014), 1311.0630.

[10] R.-A. Tripolt, L. von Smekal, and J. Wambach, Phys.Rev. D90, 074031 (2014), 1408.3512.

[11] A. Windisch, R. Alkofer, G. Haase, and M. Liebmann, Comput. Phys. Commun. 184, 109 (2013), 1205.0752.

[12] S. Strauss, C. S. Fischer, and C. Kellermann, Phys.Rev.Lett. 109, 252001 (2012), 1208.6239.

[13] A. Windisch, M. Q. Huber, and R. Alkofer, Phys.Rev. D87, 065005 (2013), 1212.2175.

[14] A. Windisch, M. Q. Huber, and R. Alkofer, PoS ConfinementX, 060 (2012), 1301.3525.

[15] A. Windisch, M. Q. Huber, and R. Alkofer, Acta Phys.Polon.Supp. 6, 887 (2013), 1304.3642.

[16] fQCD Collaboration, J. Braun, A.K. Cyrol, L. Fister, W.-J. Fu, T.K. Herbst, M. Mitter, J.M. Pawlowski, F. Rennecke, and N. Strodthoff.

[17] J.-P. Blaizot, J. M. Pawlowski, and U. Reinosa, Phys.Lett. B696, 523 (2011), 1009.6048.

[18] M. E. Carrington, W.-J. Fu, D. Pickering, and J. W. Pulver, Phys.Rev. D91, 025003 (2015), 1404.0710.

[19] J. F. Rentrop, S. G. Jakobs, and V. Meden, J. Phys. A48, 145002 (2015), 1501.00800.

[20] T. Gasenzer and J. M. Pawlowski, Phys.Lett. B670, 135 (2008).

[21] G. Baym and N. D. Mermin, Journal of Mathematical Physics 2, 232 (1961).

[22] N. Landsman and C. van Weert, Phys.Rept. 145, 141 (1987).

[23] M. Le Bellac, Thermal field theory (Cambridge University Press, 2000).

[24] C. Wetterich, Phys.Lett. B301, 90 (1993).

[25] J. M. Pawlowski, Annals Phys. 322, 2831 (2007), hepth/0512261.

[26] F. Marhauser and J. M. Pawlowski, (2008), 0812.1144.

[27] M. Buballa, K. Heckmann, and J. Wambach, Prog. Part. Nucl. Phys. 67, 348 (2012), 1202.0724.

[28] R. Lang and W. Weise, Eur. Phys. J. A50, 63 (2014), 1311.4628.

[29] R. Lang, N. Kaiser, and W. Weise, Eur. Phys. J. A51, 127 (2015), 1506.02459.

[30] S. Ghosh, T. C. Peixoto, V. Roy, F. E. Serna, and G. Krein, Phys. Rev. C93, 045205 (2016), 1507.08798.

[31] A. J. Helmboldt, J. M. Pawlowski, and N. Strodthoff, Phys.Rev. D91, 054010 (2015), 1409.8414.
[32] J. M. Pawlowski and F. Rennecke, Phys. Rev. D90, 076002 (2014), 1403.1179.

[33] L. Fister and J. M. Pawlowski, (2011), 1112.5440.

[34] L. Fister and J. M. Pawlowski, Phys. Rev. D92, 076009 (2015), 1504.05166.

[35] K.-c. Chou, Z.-b. Su, B.-l. Hao, and L. Yu, Phys.Rept. 118, 1 (1985).

[36] A. K. Das, Finite temperature field theory (World Scientific, 1997).

[37] L. Canet, B. Delamotte, O. Deloubriere, and N. Wschebor, Phys. Rev. Lett. 92, 195703 (2004), condmat/0309504.

[38] R. Gezzi, T. Pruschke, and V. Meden, Phys.Rev. B75, 045324 (2007), cond-mat/0609457.

[39] S. G. Jakobs, V. Meden, and H. Schoeller, Physical Review Letters 99, 150603 (2007), cond-mat/0702494.

[40] J. Berges and G. Hoffmeister, Nucl.Phys. B813, 383 (2009), 0809.5208.

[41] H. Schoeller, European Physical Journal Special Topics 168, 179 (2009), 0902.1449.

[42] T. Gasenzer, S. Kessler, and J. M. Pawlowski, Eur.Phys.J. C70, 423 (2010), 1003.4163.

[43] L. Canet, H. Chaté, B. Delamotte, and N. Wschebor, Physical Review Letters 104, 150601 (2010), 0905.1025.

[44] T. Kloss and P. Kopietz, Phys. Rev. B83, 205118 (2011), 1011.4943.

[45] L. Canet, H. Chaté, B. Delamotte, and N. Wschebor, Phys. Rev. E84, 061128 (2011), 1107.2289.

[46] T. Kloss, L. Canet, and N. Wschebor, Phys. Rev. E86, 051124 (2012), 1209.4650.

[47] L. M. Sieberer, S. D. Huber, E. Altman, and S. Diehl, Physical Review Letters 110, 195301 (2013), 1301.5854.

[48] D. Mesterházy, J. H. Stockemer, L. F. Palhares, and J. Berges, Phys.Rev. B88, 174301 (2013), 1307.1700.

[49] L. M. Sieberer, S. D. Huber, E. Altman, and S. Diehl, Phys. Rev. B89, 134310 (2014), 1309.7027.

[50] S. Mathey, T. Gasenzer, and J. M. Pawlowski, Phys. Rev. A92, 023635 (2015), 1405.7652.

[51] F. Reininghaus, M. Pletyukhov, and H. Schoeller, Phys. Rev. B90, 085121 (2014), 1405.3150.

[52] D. Mesterházy, J. H. Stockemer, and Y. Tanizaki, Phys. Rev. D92, 076001 (2015), 1504.07268.

[53] J. Berges and D. Mesterházy, Nucl.Phys.Proc.Suppl. 228, 37 (2012), 1204.1489.

[54] J. Berges, AIP Conf. Proc. 739, 3 (2005), hep$\mathrm{ph} / 0409233$.

[55] P. Aurenche and T. Becherrawy, Nucl.Phys. B379, 259 (1992).

[56] R. Kobes and G. Semenoff, Nucl.Phys. B260, 714 (1985).

[57] H.-H. Xu, Phys.Lett. B342, 219 (1995).

[58] D. F. Litim, Phys.Rev. D64, 105007 (2001), hepth/0103195. 